University of Nebraska - Lincoln

DigitalCommons@University of Nebraska - Lincoln

Public Access Theses and Dissertations from

Education and Human Sciences, College of

the College of Education and Human Sciences

$12-2011$

\title{
Validation of Chinese Women's Alcohol Expectancy Instrument
}

Yue Qiu Yu

University of Nebraska-Lincoln, moon1and1@gmail.com

Follow this and additional works at: https://digitalcommons.unl.edu/cehsdiss

Part of the Education Commons

Yu, Yue Qiu, "Validation of Chinese Women's Alcohol Expectancy Instrument" (2011). Public Access

Theses and Dissertations from the College of Education and Human Sciences. 131.

https://digitalcommons.unl.edu/cehsdiss/131

This Article is brought to you for free and open access by the Education and Human Sciences, College of (CEHS) at DigitalCommons@University of Nebraska - Lincoln. It has been accepted for inclusion in Public Access Theses and Dissertations from the College of Education and Human Sciences by an authorized administrator of DigitalCommons@University of Nebraska - Lincoln. 
Validation of Chinese Women's Alcohol Expectancy Instrument

by

\title{
Yue Qiu Yu
}

\author{
A Thesis \\ Presented to the Faculty of \\ The Graduate College at the University of Nebraska \\ In Partial Fulfillment of Requirements \\ For the Degree of Master of Arts
}

Major: Educational Psychology

Under the Supervision of Professor Douglas F. Kauffman

Lincoln, Nebraska

December, 2011 
Validation of Chinese Women's Alcohol Expectancy Instrument

Yue Qiu Yu, M.A.

University of Nebraska, 2011

Adviser: Douglas F. Kauffman

The present study was designed to develop and validate the Chinese Women's

Alcohol Expectancy scale; and to examine gender differences in alcohol expectancy. 134

interviewees from Canton, Kaiping and Hong Kong participated in study 1. 1,550

students from thirteen universities in three provinces, Beijing, Yunnan and Wuhan, China, completed the women's alcohol expectancy questionnaire in study 2 . The findings in study 1 revealed six prominent categories that inform women's alcohol expectancy. Findings in study 2 provided 7-subscales $(\alpha>0.7)$ for the instrument's reliability and validity. Additionally, there were significant differences in alcohol expectancy by genders. Implications related to expectancy theory and gender is discussed. 


\section{ACKNOWLEDGMENTS}

The present study is a collective work involving my UNL professors, colleagues, and friends from the United States and China (Kaiping, Hong Kong and Canton city). I thank my former students and friends in China that helped me make social connections while conducting my study. I am grateful for the help and support of all my friends, family and professors in Kansas, Lincoln and China, and their contributions to the present study.

I thank my adviser Dr. Doug F. Kauffman, Dr. Ian Newman, and Dr. Duane Shell, for their encouragement and support in conducting this study and writing this thesis. A thank you to Jim and Carol who provided me a country house in which to write. A thank you to my friend and colleague, Andrea Nickel, who edited the final version of this study. Finally, a special thank you goes to my dearest mother Mei Ling, for her prayers, loving and caring linger in my life, and have made this study possible. 


\section{Table of Contents}

Chapter 1 - Significance of the Study and Research Questions.........................5

Expectancy Theory ...........................................................................................6

Expectancy theory relates to motivation...............................................6

Historical views. ..........................................................................7

Other views. ...............................................................................................................8

Expectancy Theory Application............................................................9

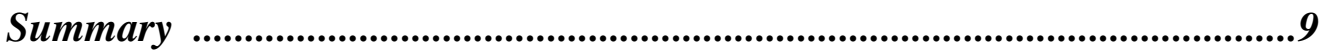

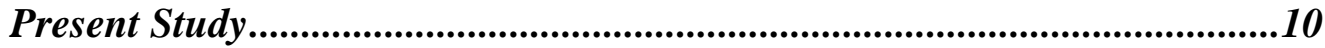

Identification of a risk health behavior...................................................10

Significance of the Study .................................................................11

Research Question ........................................................................................12

Chapter 2 - Literature Review ....................................................................15

Expectancy Theory ..................................................................................15

Bolles' S-S* and R-S* expectancy models ................................................16

Bandura's outcome expectancy theory. .............................................16

Achievement studies on expectancy theory. ...........................................18

Health studies on expectancy theory..........................................................20

Expectancy correlates a behavior. .................................................21

Expectancy predicts future onset of a behavior.................................23

Expectancy mediates a behavior and its outcome...............................24

Alcohol Use Issues of Chinese Women ...................................................227

Application of Expectancy Theory to Women's Alcohol Use .......................29

Women's alcohol expectancy. ........................................................................29

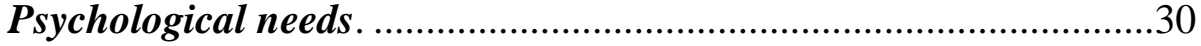

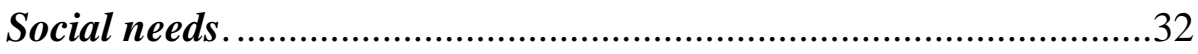

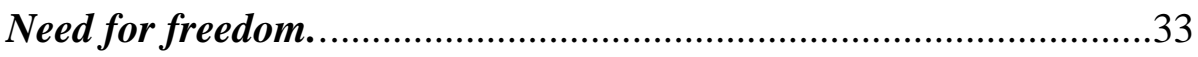

Men and women's alcohol expectancy.....................................................35

Men's and Women's Alcohol Expectancy in the West ..................................35

Men's and Women's Alcohol Expectancy in China......................................37 
Chapter 3 - Study 1 Method .........................................................................................40

Participants................................................................................................40

Materials …...............................................................................................41

Semi structural interview questions. .............................................................41

Procedure ....................................................................................................42

Interview place selections. ..............................................................................42

Interview transcribing steps................................................................................43

Chapter 4 - Study 1 Results......................................................................................44

Discussion..................................................................................................44

Chapter 5 - Study 2 Method ............................................................................................50

Items Development ............................................................................................

Participants.............................................................................................55

Materials ………….......................................................................................55

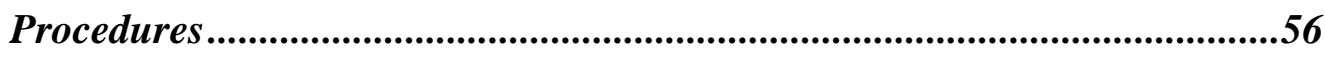

Survey place selections. .............................................................................56

Data analysis. .............................................................................................57

Chapter 6 - Study 2 Results .........................................................................................58

Validation of Chinese Women's Alcohol Expectancy Instrument ................58

Expectancy differences between Chinese men and women ...........................68

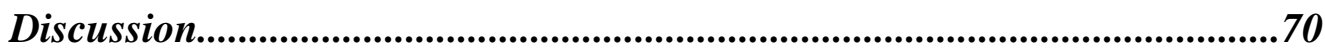

Chapter 7 - Overall Discussion ........................................................................................73

Major Findings ............................................................................................73

Convergent findings..........................................................................................73

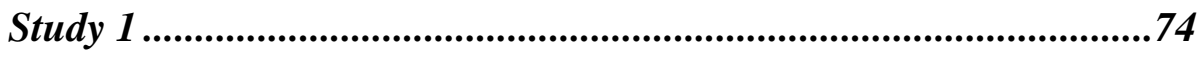

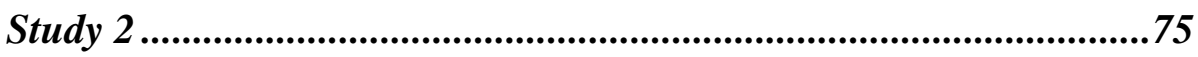

Divergent Findings.................................................................................................76

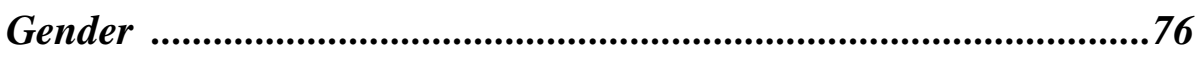

Implications .....................................................................................................77

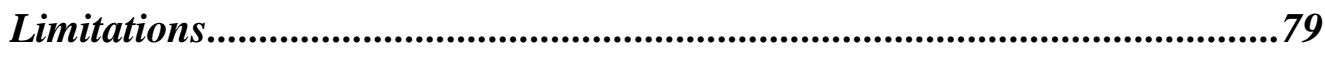

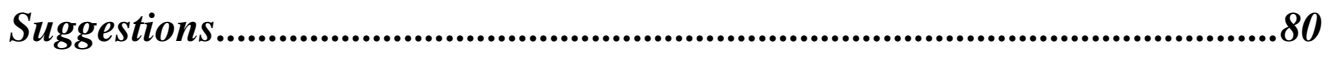

BIBLIOGRAPHY .............................................................................................................1 


\section{List of Tables}

Table 1 Preliminary Data: Themes ............................................................51

Table 2 Similarities and Differences between Themes and 7-Factors..............61

Table 3 Chinese Women's Alcohol Expectancy Items and Factor

Loading for the Final Instrument Items and Scales ........................63

Table 4 Chinese Women's Alcohol Expectancy Items and Factor

Loading for Males Final Instrument Items and Scales ....................65

Table 5 Chinese Women's Alcohol Expectancy Items and Factor

Loading for Females Final Instrument Items and Scales..................67

Table 6 Students' Mean Scores on Chinese Women's Alcohol

Expectancy by Gender 


\section{List of Appendices}

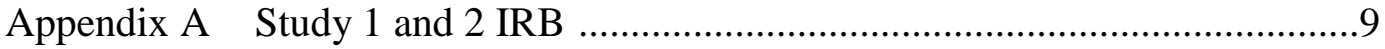

Appendix B Pilot Study - Interview Samples ...............................................13

Appendix C Samples of Women's Alcohol Expectancy..................................15

Appendix D 96-items Alcohol Expectancy Statements ..................................21

Appendix E Chinese Women's Alcohol Expectancy Instrument ....................25

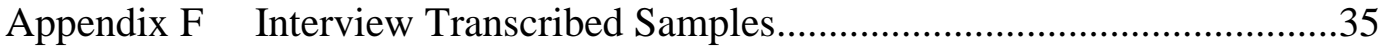




\section{Chapter 1}

\section{Significance of the Study and Research Questions}

Educational psychologists' research in the areas of cognition and motivation aims toward the development of improved educational practices. It focuses on increasing the understanding of learning processes, in order to promote the advancement of education, and also the understanding of learners' motivations to engage in behaviors, and how this affects their cognition. Individuals set specific expectations for learning content, which may guide them to behave and to regulate motivation differently. For instance, many researchers in modern educational psychology have been addressing the fundamental idea that expectations of beliefs or goals for learning outcomes form a salient power to motivate students to learn, and is a major aspect of motivation (Bolles, 1972; Bandura, 1977; 2004; Ames, 1992; Wigfield, Tonks and Eccles, 2004; Lambright, 2010). Understanding a student's particular behavior first requires a definition of the theoretical basis for motivation.

Bandura (1977) proposed outcome expectancy theory as part of his social cognitive theory. Outcome expectancy theory provides one explanation for why individuals perform particular behaviors and the ways in which individuals learn about behaviors before they have performed them. Simply stated, expectancy knowledge motivates future behavior. In the present study, I will briefly review how motivation is related to expectancy theory and describe expectancy theory, and finally address studies related to the theory's application. 


\section{Expectancy Theory}

\section{Expectancy Theory Relates to Motivation}

Researchers have worked for decades to be able to describe how human motivation and cognition relate to expectancy theory. Motivation in modern psychology has been well defined as an energizing action related to beliefs, values and goals. As it relates to beliefs or goals, this motivational phenomenon is called an expectancy. Eccles and Wigfield (2002; 2004) described the underlying meaning of an expectancy as an individual's belief about what outcomes are achievable through performing a designated behavior. These expectancies act as motivators for individuals who intend on performing a behavior in order to achieve its outcomes. Expectancy theory serves as a cognitive process and motivational phenomenon for particular tasks (Bolles, 1972; Bandura, 1977; Smith \& Goldman, 1994; Eccles and Wigfield, 2002; Wigfield, Tonks, and Eccles, 2004; Lambright, 2010; Moss and Albery, 2010).

\section{Historical Views}

Bandura and Bolles are theorists who have discussed expectancies within the motivational phase and its role as a cognitive process and motivational phenomenon. Bolles' (1972) work, later supported by Bandura (1977), described the ways that human motivation and cognition work under outcome expectancy theory. Motivational expectations will be explored in the current study.

In early expectancy development work, Bolles (1972) explained the basic concept of expectancy theory in motivation in the expectancy-response model. Tollman's 1932 work on the learning effect law described how individuals receive stimuli, and what individuals learn about the stimuli, which is derived from their self-expectancy $\left(\mathrm{S}^{*}\right)$. 
Expectancy theory can also explain an outcome within motivation and cognition. Expanding on Bolles' (1972) work, Bandura (1977) proposed outcome expectancy theory in his social cognitive theory. Outcome expectancy theory provides one explanation for why individuals perform certain behaviors, and the ways in which individuals learn about behaviors before they perceive the behaviors. Outcome expectancy refers to individuals' evaluation of pieces of information about the outcome of a certain behavior before they execute that behavior.

\section{Other Views}

Educational psychologists and educators adapted classical expectancy theory in order to define learners' or individuals' motivation related to a particular behavior and its outcome, and tried to determine individuals' motivational aspects, such as expectations of perceiving a behavior (Heys, 1985; Maiston, Carey, and Bradizza, 1999; Wigfield, Tonks and Eccles, 2004). A key component of Wigfield, Tonks and Eccles' (2004) cultural value expectancy model was utility task value, which is consistent with the ideas that have been proposed in Bolles' and Bandura's expectancy work. Utility task value refers to an individual's opinion of the value of a task, or how much the individual values the probability of usefulness by attaining the task. For example, a person chooses to take a biology class, because he valued its usefulness to fit his or her plan, which is to complete a pre-med major and to enter medical school in the future.

In closing, expectancy is a contingency representation between a behavior and its outcome. This concept indicates individuals' expectations of beliefs or goals on a behavioral outcome. Bolles' S-S* and R-S* expectancy model and Bandura's outcome expectancy theory provide ideas for why individuals perform particular behaviors. 
Individuals expect the outcomes of a behavior before they perform the behavior. Briefly, individuals' present motivation outcomes are related to a target behavior in mind before perceiving a behavior. For instance, a person has stored an expected outcome of a behavior in memory (i.e. if I study hard, I will keep up my GPA) before the individual performs the behavior (Smith and Goldman, 1994). This can influence thoughts about the behavior, and whether or not the individual decides to perform it. Overall, expectancy theory springs from a cognitive learning process and it is a concept in motivation.

\section{Expectancy Theory Application}

Expectancy theory has served in multiple practical settings, such as achievement studies, public professional services, health education and prevention studies (Batlis and Waters, 1973; Lambright, 2010; Schwargzer and Fuchs, 1995; Smith \& Goldman, 1994; Bandura, 2004; Stacy, 1990; 1997; Newman, I. M., Qian, L., Shell, D. F., Qu, M., \& Zhang, Y., 2006; Shell, D. F., Newman, I. M., \& Qu, M., 2009; 2010). In the school achievement studies, researchers indicated that individuals' outcome expectancy on certain learning content affected their motivation and performance (Hays, 1985; Wigfield et al., 2004). As another example, Wigfield et al. (2004) proposed the expectancy value model, which indicated that an individual's success is influenced through his or her value of experiences and cultural aspects, and found that expectancy strongly predicted their achievement outcomes and task involvement. Lambright (2010) studied expectancy theory and found contracted providers were motivated to use service-monitoring tools correctly because of the rewards (bonuses) and penalties (dismissal) associated with performing the task correctly or incorrectly. 
In health education and prevention research studies, previous researchers adapted expectancy theory sufficiently in order to understand the motivational reasons why people behave or act in certain harmful health behaviors, such as smoking, obsessive alcohol consumption, or taking drugs. For example, a cardiovascular disease prevention program leveraged an individual's behavioral outcome expectancy, and helped an individual with perceived motivation to adopt better health habits (such as quitting smoking or obsessive drinking). Bartholow, B. D., Sher, K. J., and Strathman, A. (2010) found a linear function that related outcome expectancies and alcohol use for college students with high expectations of alcohol consumption for all four years. A study from Smith and Goldman (1994) found that early adolescents did not perform obsessive drinking behaviors until they were later adolescents. Shell et al., (2009; 2010) indicated that positive alcohol expectancies had more of an effect on alcohol consumption. Overall, expectancies predicted the future onset of adolescents' alcohol consumption-related problems and how expectancies were linked to drinking behaviors. This contributes to public health professionals' knowledge of individuals' motivational levels in perceiving healthy behaviors (Stacy, A.W., Widaman, K. F. \& Marlatt., 1990; Stacy, A. W., 1997; Schwargzer and Fuchs, 1995).

\section{Summary}

Previous research studies addressed important features of understanding learners' motivations to engage in behaviors, and in perceiving particular behaviors. Health education studies, for the most part, have adapted expectancy theory efficiently. For instance, Davis, K. C., Norris, J., Hessler, D. M., Zawacki, T., Morrison, D. M. and George, W. H. (2010) indicated underage women's drinking behaviors were indirectly 
mediated by their sexual cognitive intentions, increasing unprotected sexual behavior. Donovan, J. E., Molina, B. S. G, and Kelly, T. M. (2009) found negative alcohol expectancies did not protect children from alcohol consumption as they grew older. These findings were consistent with Edwards' (1973) and Stacy et al.'s (1990) studies. Educational psychologists and health professionals have examined the effectiveness of expectancy models of alcohol consumption by comparing attitude models of alcohol use.

\section{Present Study}

\section{Identification of a Health Risk Behavior}

Expectancy theory provides a way to understand what motivates people's behaviors. Expectations that a certain behavior will lead to an outcome that individuals value or devalue will motivate an individual to perform or not to perform the behavior. The present study asks the question of what current health issue affecting human quality of life could benefit from a practical application of expectancy theory.

In recent decades, the global misuse of alcohol has affected the quality of human life. Alcohol consumption is increasing worldwide, with associated human health risk factors. In 2009, $9 \%$ of worldwide deaths $(320,000)$ for young people between the ages of 15-19 were due to alcohol-related public health problems such as traffic injuries, mental disorders, fatty liver diseases, digestive system disease, hepatitis, and unprotected sexual behavior (Kim, J. H., Chan, K. W. C., Chow, J. K.W., Fung, K. P., Fong, Y. F., Cheuk, K. K. and Griffiths, S. M., 2009; Giang, K.B., Allebeck, P., Spak, F., Minh, H. V. and Dzung, T. V., 2008; WHO, 2009; WHO, 2004; Cochrane, J., Chen, H., Conigrave, K. M., and Hao, W., 2003). One striking research study indicated alcohol production in China increased $0.4 \mathrm{Kg}$ ethanol per person in $1952 ; 2.5 \mathrm{~kg}$ ethanol per person in $1978 ; 22.9 \mathrm{~kg}$ 
ethanol per person in 1997; and 42.5kg ethanol per person in 2005 (Hao and Young, 2000; Cochrane et al., 2003; Hao, W., Chen, H., and Su, Z., 2005). Additionally, the rate of alcohol consumption for urban Chinese women aged 18 or older increased from $34 \%$ to $63 \%$ from 2002-2005 (Zhang, J., Casswell, S., and Cai, H., 2008; Li,J., Ma, W., Xu, Y., 2006; Hao et al., 2005; and Ma, G., Zhu, D., Hu, X., Luan, D., Kong, L., and Yang, X., 2005).

Statistical information about Chinese alcohol consumption may present a reason for concern about the onset of public health problems in China. For 7000 years, wine consumption has historically been viewed by Chinese society as daily food, assumed to pose few health hazards for the population (Lu, Z. P., Engs, R. and Hanson, D., 1997). The assumption seems overly optimistic for present-day China. The World Health Organization (WHO) (2004) reported half of all automobile crashes $(616,971)$ in China in 2000 involved drunk drivers, resulting in 98,853 total deaths. Increased alcohol consumption among the Chinese population, particularly among women, could lead to potential public concerns for Chinese society. Alcohol-dependent females suffered increased physical impairment and worse general health than alcohol-dependent males (Peter, T. J., Millward, L. M. and Foster, J., 2003). Thus, it is important to be aware of the use of alcohol by Chinese women as a health issue in China. Chinese women's motivations to consume alcohol appear to be reflected in memory by expectancy.

\section{Significance of the Study}

A careful review of these studies gives rise to a consideration of the influence of motivation on Chinese women when they choose to consume alcohol (Xu, 2003; Shu, 2004; He, 2004; Wang, 2008; Huo, 2001; Newman et al., 2006; Shell et al., 2009; 2010; 
Bryant, 2000; Lyons, A.C., Dalton, S. I. and Hoy, A., 2006). Some studies propose that exaggerated economic growth and changing social roles with increased social freedom for Chinese women may be factors influencing the narrowed gap between alcohol consumption trends of men and women (Hao and Young, 2000; Cochrane et al., 2003; Hao, et al., 2004). Expectancy theory can be adapted to clearly view the outcomes of that decision-making and to observe the obsessive drinking behavior among individuals (Christiansen, B. A., Goldman, M. S., and Inn, A., 1982; Smith \& Goldman, 1994; Stacy et al., 1990; 1997). Thus, applying expectancy theory to the present study will help describe motivational influences surrounding Chinese women's alcohol-related behaviors: their alcohol expectancy.

The mixed method designs of the empirical studies into the reasons behind the increase in alcohol consumption by women in China were limited. In addition, the Shell et al.'s alcohol expectancy instrument has not clearly defined the differences in motivational level behind alcohol consumption between males and females (Newman et al., 2006; Shell et al., 2009; 2010). Based on these limited criteria, such as Shell et al.'s alcohol expectancy scales, there is a need to understand women's expectations of alcohol consumption in the literature, and to conduct a study to develop and validate an instrument to measure women's alcohol expectancy. This will provide a tool which may be used for alcohol prevention or intervention strategies.

\section{Research Question}

The WHO reports and empirical studies are evidence that Chinese women's alcohol consumption should be a matter of concern. Other qualitative studies in the western literature showed that women's expectations of alcohol consumption were 
different from those of men (Lyons and Willott, 2008; Edwards, G., Hensman, C. and Peto, J., 1973; Cooper, M. L., Russell, M., Skinner, J. B., Fromme, M. R., and Mudar, P., 1992; Rauch and Bryant, 2000). However, there are no systematic measurement scales for measuring women's alcohol expectancies. In addition, there are no studies targeting Chinese women's motivational aspects of alcohol consumption, so the research question of what Chinese women's expectations of alcohol consumption are remains unanswered. Some argument might arise as to how exactly to develop a motivational scale related to Chinese women's alcohol consumption expectations; as the scale is one-dimensional and distinct from a measurement of the differences between men and women's alcohol expectancy. In the past 20 years researchers, such as Smith \& Goldman (1994), Shell et al., (2009), Shell et al., (2010) and Newman et al., (2006) have compared gender differences and found no significant differences in alcohol expectancy. In these cases, an appropriate method for measuring gender differences may not have been used. For example, participants of each gender were asked separately about what alcohol expectancies were, and survey items were made based on both men's and women's perceptions of alcohol consumption. However, conceptual differences in alcohol expectancies may vary by gender, and the present study aims to develop a better approach to assessing gender differences based on this belief.

The development of the instrument will be established in study 1 and the validation of the instrument and examination of alcohol expectancy by gender are conducted in study 2 . Thus, the purpose of this study is twofold: 1) develop and validate an instrument related to Chinese women's alcohol expectancy through mixed methods; and 2) examine the Chinese women's alcohol expectancy instrument to determine 
whether there is a significant difference between men's and women's expectations of alcohol consumption. 


\section{CHAPTER TWO}

\section{Literature Review}

Previous studies have shown that individuals' self-expectancy stems from longterm memories of outcome expectancies (Bolles, 1972; Bandura, 1977; Smith \&

Goldman, 1994). This theory can be adapted to study outcome expectancy and decisionmaking factors surrounding obsessive drinking behaviors (Stacy, 1990; 1997; Smith \& Goldman, 1994; Bandura, 2004), and can provide a way to understand the underlying motivation of Chinese women's future alcohol behaviors (Shell et al., 2009; 2010; and Newman et al., 2006).

First, I will review the innate meaning of expectancy theory. Secondly, I will show how empirical studies have related expectancy theory to human behavior. Thirdly, behavioral research on women's alcohol expectancies and motivation will be examined. Finally, studies linking the alcohol consumption expectations of men and women will be discussed.

\section{Expectancy Theory}

To understand a particular behavior, one first needs to understand the motivation behind that behavior. Expectancy theory involves a behavioral outcome and refers to how individuals perceive behaviors based on prior outcomes of those behaviors. The fundamental concept of an expectancy is a representation between a behavior and its future outcome (Bolles, 1972; Bandura, 1977; Smith \& Goldman, 1994).

\section{Bolles' $S$-S* and R-S* Expectancy Models}

In his early expectancy development work, Bolles argued that a learned behavior under operant conditioning involves two kinds of alternative reinforcements 
(motivations): an incentive motivation and a cognitive approach that indicate what individuals have learned. He challenged other researchers to rethink the pure reinforcement (motivation) that does not fully explain what an individual learns in many cases. Bolles argued that the pure reinforcement of superstitions fails. Superstitious behavior refers to a behavior that occurs right after a reinforcer. Bolles addressed an example, and informed involving food and a press-paddle indicated that reinforcement could only direct toward a behavior by providing food, and could not specify whether individuals were waiting or performing the press-paddle behavior during the interval time (before the reinforcer appeared).

Bolles' work brought attention to how expectancies were involved in motivation and cognition. A reinforcement was the motivational effect. Individuals with a feedback incentive would be motivated to reach the destination of learned behavior. Individuals not only could learn to respond with a behavior, but also could learn what to expect through reinforcement. Therefore, a combination of the S-S* (stimuli - self-expectancy) and R-S* (response - self-expectancy) models could explain a consequence behavior: how individuals are motivated to engage in a behavior and how responses correspond to, or are derived from, prior expectancy outcomes.

\section{Bandura's Outcome Expectancy Theory}

Bandura (1977) stated four factors that influence the human cognitive process: performance accomplishments, vicarious experiences, verbal persuasion and physiological states. He suggested that behavioral changes were a state of presentation of cognition. In other words, changes or responses in behavior are not directly connected to 
the influence of stimuli; instead, individuals analyze and combine pieces of information about the outcomes of their behavioral responses to the stimuli.

Bandura described how the four factors influence individual levels of motivational change in behaviors. The performance accomplishment factor refers to how continued performance of a behavior is related to, or depends on, an increasing number of personal successful experiences with the behavior. An individual's outcome expectancies derived from their successful or valuable experiences of a behavior's effects will make the same behavior occur again. A vicarious experience occurs when, instead of an individual mastering to-be-learned information on his or her own, others are observed performing a target behavior successfully or unsuccessfully. Individuals can generate behavioral outcome expectancies from observing others' behaviors, and increase their confidence in performing the behaviors themselves.

Verbal persuasion refers to talking aloud to encourage a behavior, which helps an individual maintain focus on performing a designated behavior. This factor describes how an individual's beliefs have an effect on his or her performing a behavior based on his or her expectations of the behavioral outcomes. With increased successful experience, individuals will also eventually increase confidence. Emotional arousal can also influence an individual's motivational level: high arousal will inhibit motivation, and low arousal will augment it. Positive outcome expectancy increases individual motivation to perform a behavior; negative outcome expectancy has the opposite effect.

The concept of outcome expectancies is based on these four factors. A behavior will not be executed until an individual verifies information about the outcome. There are two levels of perceived self-efficacy that relate to particular outcomes. If a person 
devalues the outcomes of to-be-learned behavioral information, he/she will not execute the behavior; however, if the information about the outcome is valued, they will engage in the behavior. According to Bandura (1977), individuals' expectations about the outcome of a behavior would not influence their choice of behavior. Rather, the person would value information about the outcome of a behavior that comes from performing the behavior and reaching the outcome.

In summary, Bandura's outcome expectancy provides one explanation for describing motivation, as it relates to cognition. As individuals think about the outcomes they wish to achieve, they may recall a past behavior that resulted in a positive or negative outcome, and will use this to inform their target behavior for achieving their new desired outcome.

\section{Achievement Studies on Expectancy Theory}

Applying the expectancy theory concept, Wigfield, Tonks and Eccles (2004) looked at expectancy for achievement and proposed a utility task value concept, which was similar to Bandura's outcome expectancies work. This concept says that students' beliefs regarding the performing of a task which fulfills their future goals or plans strongly predicts their achievement outcomes and task involvement. For instance, Wigfield et al. found a negative trend in terms of perspectives on expectancy and values of learning among primary and secondary school children. They observed that children might not find value in performing a task because of that task's lack of usefulness to their goals. The task value expectancy models can fairly specifically explain expectancies that students develop in life and that motivate their future behaviors (Smith \& Goldman, 1994; Shell et al., 2009; 2010; Newman et al., 2006). 
Expectancy theory has also been adapted to public services. Lambright's (2010) instrumental expectancy focused on the extent to which expectancy theory relates to motivation. Consistent with Bandura's and other researchers work, Lambright's main concept states that motivation is a function of expectancy, instrumentality, and valence, and complicated beliefs can lead to varying motivations. Lambright described three attitudes of motivation: expectancy, instrumentality, and valence. Expectancy refers to the belief an individual has about his/her ability to perform a task before that task is performed. The individual also has a belief regarding the outcome of a performed task, and instrumentality refers to the connection between the beliefs in ability and the performance outcome. Valence is the process in which the individual assigns value (satisfaction or dissatisfaction) to a performance outcome.

Lambright (2010) used a qualitative approach and developed an insightful systematic understanding of expectancy theory related to the complexity of motivational level among contracted providers at governmental worksites. She examined the motivation levels of contracted public service providers with relation to service regulations. The finding was that over $60 \%$ of the time, contracted providers were motivated to use service-monitoring tools correctly because of the rewards (bonuses) and penalties (dismissal) associated with performing the task correctly or incorrectly. Lambright's (2010) finding was similar to the previous studies on expectancy utility task value related to motivation (Eccles and Wigfield, 2002; Wigfield, Tonks and Eccles, 2004). These studies have explained how to predict an individual's motivations and behavior in the future, and indicated what role expectancy plays in influencing an individual's motivation to engage in a behavior. 


\section{Health Studies on Expectancy Theory}

Health education research can be refined through relevant expectancy work, such as alcohol expectancy. Alcohol expectancies served three motivational phases (Edwards, 1973; Smith \& Goldman, 1994; Christiansen et al., 1982; Stacy, 1997; Agrawal, A., Dick, D. M., Bucholz, K. K., Madden, P. A. F., Cooper, M. L., Sher, K. J. and Heath, A. C., 2007; Bartholow et al., 2010). Expectancy Correlates with a Behavior (alcohol consumption)

The primary motivational phase of expectancy and alcohol use discovered a relationship between an expectancy and a behavior. For example, applying Bolles' (1972) S-S*and R-S* models to a person observing and learning about a drinking behavior helped determine that the person stored these drinking behaviors in memory. Later, the person derived from his/her memories the outcomes of the drinking behavior. If he/she valued the outcomes, he/she then responded by consuming alcohol. If he/she did not value it, the drinking behavior might not occur. Similar application of Bandura's (1977) outcome expectancy theory and Wigfield and Eccles' (2004) utility task value concept supported the observation that if a person values alcohol consumption as a behavior that helps them release anger, he/she will be more likely to drink. On the other hand, if he/she believes that drinking could ruin his/her reputation, he/she might not be as likely to perform drinking behaviors. The application of expectancy theory to alcohol use behaviors is an important part of defining the reasons why individuals' certain behaviors are motivated to occur (Christiansen et al., 1982; Smith \& Goldman, 1994; Newman et al., 2006; Shell et al., 2009; 2010). 
Research studies have predicted the relationship between an expectancy and a behavior. In addition, these studies examined whether individuals developed expectations of alcohol consumption correlating with the alcohol consumption behavior. For example, Christiansen et al. (1982) examined when adolescents' alcohol expectancies were formed, and whether from pharmacological or social-learning factors. 1,580 students (age range 12-19) from Detroit school districts participated in a 10-year longitudinal study in which researchers observed their drinking behavior until the students turned age 29. The instrument that researchers used was the AEQ-A (Alcohol Expectancy QuestionnaireAdolescents, Brown, 1980). Researchers divided participants into three age groups and determined students' alcohol expectancy factors. There were six expectancy factors: physical tension reduction, worry reduction, modification of social and emotional behaviors, power, and pleasure. Participants were divided into three classes: lowermiddle-class, middle-class and upper-middle-class.

Their findings indicated the alcohol expectancies that preadolescents developed were influenced by parents' drinking behaviors, observations of others' drinking, and peers. As students' ages increased and they gained more drinking experiences, their expectations of seeking alcohol consumption increased. Pharmacological experiences influenced adolescents' alcohol expectancy as well. From Christiansen et al.'s (1982) study, adolescents seemed to develop their alcohol expectancy at a very young age through environmental influences, such as their parents, peers and their own drinking experiences.

Expectancy was found in these studies to have significant correlation with the alcohol behavior. Bartholow et al. (2010) investigated possible individual differences in 
the relationship between alcohol expectancy and alcohol use. They indicated that selfconsciousness could have a great impact on directing behaviors: self-consciousness refers to an individual's stable motives and attention, and whether the individual is aware of his or her beliefs. 3,156 Midwestern University freshmen participated in a four-year longitudinal study. Participants completed questionnaires and were interviewed about their alcohol use, outcome expectancy and personality. Researchers also used positive expectancy regarding the effects of the alcohol questionnaires. The study found that a linear function related outcome expectancy and alcohol use for students with high expectations of alcohol consumption for all four years. Students in the first and second years were under the legal drinking age, and that social environmental cue could affect students' future alcohol use. Adolescents seemed to develop their alcohol expectancy at a very young age through environmental influences, such as their parents, peers and their own drinking experiences. These experiences were indicated in Bandura's (1977) outcome expectancy theory through concepts such as vicarious learning experiences and accomplishments. Expectancy seems to have a significant effect on human decisionmaking and thoughts on alcohol consumption. This knowledge provides information, which shows the reasons why college students decide to drink obsessively or in moderation.

Christiansen et al.'s (1982) and Bartholow et al.'s (2010) studies demonstrate that individuals developed a series of expectancies related to a behavior (alcohol consumption). They used a systematic measurement scale to examine these relationships. Similar findings were also presented in later studies (Goldman et al., 1982; Stacy, 1990; 1997; Agrawal et al., 2007; Donovan et al., 2009). 


\section{Expectancy Predicts Future Onset of a Behavior (alcohol consumption)}

The second motivational phase of expectancy showed how expectancy could predict the future onset of a behavior. Outcome expectancy and sensation-seeking were independent predictors for future use. Smith and Goldman (1994) presented a 3-year longitudinal experimental study of alcohol expectancy. The purpose of their study was to identify obsessive alcohol consumption in seventh graders through alcohol expectancy theory. Research questions focused on how expectancy predicted the future onset of adolescent alcohol consumption-related problems and how expectancy was linked to drinking behaviors.

Findings from Smith and Goldman's study showed that there was a 50\% probability of student non-drinkers in the first and second years consuming alcohol in their third year. Additionally, 25\% of third year student drinkers had experiences with drunkenness, and the number of times they got drunk was two or four times more in their third year. Smith and Goldman indicated that early adolescents did not perform obsessive drinking behaviors until they were later adolescents. They used qualitative and quantitative mixed method to develop a systematic scale of alcohol expectancy in a longitudinal study. The researchers also identified individuals' expectancy, which related to the prediction of one's future drinking behavior. Donovan et al. (2009), Shell et al. (2009) and Cranford, J A., Zucker, R. A., Jester, J. M., Puttler, L. I., and Fizgerald, H.E. (2010) found results consistent with Smith \& Goldman's (1994) study. These findings showed expectancy predicted individuals' alcohol consumption. These researchers pointed out that positive expectancy could greatly influence the onset of future alcohol consumption. 


\section{Expectancy Mediates a Representation between a Behavior and its Outcome}

Expectancy could mediate individuals' alcohol use experiences and predict future use (Stacy, 1997). For instance, individuals' expectancies of the outcomes of alcohol use, such as relaxation or the release of anger, would affect motivation and influence individuals' decisions on whether or not to drink. Expectancy can be positive. A person may believe that drinking alcohol reduces aversive emotions, or facilitates socialization. Expectancy can be negative, as with a person who believes that alcohol consumption is bad for health or causes liver cancer. Previous empirical studies support this mediation of thought, using expectancy theory to demonstrate that positive expectancy outcomes predicted more use/misuse of alcohol than did negative alcohol expectancy outcomes.

Stacy et al. (1990) examined whether positive expectancy would be a better predictor of alcohol consumption than negative expectancy and attitudes. The study was guided by the alcohol expectancy theory of Goldman et al. (1980), which explained how individuals' drinking behaviors and their expectations for alcohol use and misuse were influenced. Stacy et al.'s second study, they evaluated the alcohol use expectancy model by measuring both positive and negative outcome expectancy in responses from 76 male undergraduates. They showed that a negative alcohol expectancy outcome did not significantly decrease use, but the lack of positive or negative expectancy did decrease consumption. They concluded that positive expectancy did predict alcohol use.

Stacy et al.'s third study, 56 male and female undergraduates completed measurement scales of their attitude on alcohol use and positive and negative alcohol expectancy. Positive alcohol expectancy was shown to directly predict future alcohol use or misuse, more so than negative expectancy. The authors suggested that future 
researchers could not dismiss individuals' negative alcohol expectancy because both types of expectancy in the model accounted for variance in alcohol use (35\%). Removing negative expectancy from the model reduced the variance to below $0.01 \%$. Also, both positive and negative expectancy predicted individuals' attitudes on alcohol use. Expectancy mediated individual's motivation toward alcohol use behaviors.

Donovan et al. (2009) approached outcome expectancy as a social belief phenomenon, also showing that expectancy correlated with behaviors and predicted the onset of a behavior. The study used alcohol expectancy measures adopted from various empirical studies, such as Goldman and Brown's alcohol expectancy. A 7-year longitudinal study of 452 families looked at how children learned vicariously about alcohol use. Researchers focused on how parents influenced drinking expectancy in early childhood, and found that parents' sensual or drinking experiences did influence children's drinking behavior expectancy. However, a negative relationship between age and alcohol expectancy was found after the child participants' ages were 12 or older. Participants at ages 8.5-11.5 had high negative alcohol expectancy, and drank less at that period, but their drinking behaviors increased.

Donovan et al. addressed the question of why the negative expectancy of young individuals did not have as proactive an effect for individuals who became adults, theorizing that it might be because of the influences of the media and of friends. The conclusion was that alcohol expectancy and parental involvement were social factors influencing young children and their future alcohol use. When children got older, their negative alcohol expectancy did not protect them from alcohol consumption. 
The following study by Cranford et al. (2010) indicated a similar result as Donovan et al.'s (2009) study. Adolescents' positive outcome expectancy especially influenced their future obsessive or binge alcohol drinking. Positive outcome expectancy could explain the reasons why individuals engage in binge drinking. Cranford et al. (2010) examined the relationship between adolescent drinking behaviors and alcohol expectancy. The researchers stated that parental involvement in the family mediated their child's alcohol expectancy and future alcohol use. The finding showed positive and negative alcohol-related expectancy was strongly associated with predictions of alcohol drinking behaviors. However, positive expectancy predicted alcohol drinking behaviors better than negative expectancy. The results also might indicate that adolescents' alcohol expectancy moved from more negative to positive during the period from middle childhood to early adolescence.

These findings were consistent with a previous study by Smith \& Goldman (1994). Cranford et al.'s (2010) study inferred that an individual's expectations of alcohol consumption were mediated by the influence of an individual's surrounding social factors. Other research studies, such as Shell et al. $(2009 ; 2010)$ specified positive alcohol expectancy appeared to predict individuals' alcohol consumption better than negative expectancy.

In closing, research studies relating to alcohol consumption and alcohol expectancy have dealt with beliefs about the effects of alcohol consumption and emphasized a contingency representation between a behavior and its outcome. Researchers in previous studies addressed issues or problems in society and established a strong practical framework through expectancies work, which can be used to understand 
an individual's motivation in alcohol use. These findings have great implications for the development of future health educational programs or prevention. Examples of these are informing educational psychologists and educators about the three phases of alcohol expectancy application: correlation with a behavior, prediction of the onset of a behavior, and mediation between a behavior and its outcome. Applying alcohol expectancy will also help the present work to define the reasons why Chinese women might use alcohol obsessively or moderately in modern China.

\section{Alcohol Use Issues of Chinese Women}

Previous studies have indicated that Chinese women's alcohol consumption is on the rise in China (WHO, 2004; Hao et al., 2004; Su, Z., Hao, W., and Chen, H., 2003; Wei et al., 2002; Wu, 2006; Wu, B., Mao, Z. F., Rockett, I., and Yue, Y.W., 2008; Xing, Y., Ji, C., and Zhang, L., 2006; Yan, 2007; Zhang et al., 2008), and is creating health concerns. One overwhelming trend is that more and more young high school and college age females drink alcohol in China. For example, a one-year WHO sponsored survey study by Hao et al. (2004) reported that within a sample of 24,992 community residents, $59 \%$ of the women were drinkers, and their drinking rate had increased by $10 \%$ since 2002. The gap between alcohol consumption trends of men and women has narrowed. The following studies on the drinking habits of women in China reveal a new pattern.

WHO (2004) reported a 2001 survey of 24,992 participants across five provinces in China that showed the alcohol drinking rate for men was 13.4 times that of Chinese women. A 2003 World Health Organization report showed that Chinese women consumed $20 \mathrm{~g}$ or more of pure alcohol per day, and Chinese men drank $40 \mathrm{~g}$ of pure alcohol per day (in a sample of 1,936 residents). In Ma, G., Zhu, D., Hu, X., Luan, D., 
Kong, L., and Yang, X. (2005) researchers conducted a survey study with 159,117 Chinese residents (ages 15 and older, 76,128 males and 82,989 females). They reported that $58.2 \%$ of male residents were current drinkers who consumed $100-150 \mathrm{~g}$ of spirits, and $77.3 \%$ of female drinkers consumed $50-100 \mathrm{~g}$ of spirits. The age at which people started to drink among peers was 18 or younger.

Wu et al. (2008) examined the ways that socioeconomic status (SES) influenced residents' alcohol consumption in Hubei. 15,609 Hubei residents (11,311 rural residents and 4,298 urban residents, ages 15-101 years old) completed the Chinese National Health and Nutrition Examination Survey. The dependent variable was regular alcohol consumption (how often residents consume alcohol). Independent variables were SES, education level, household income, occupational status and wealth. Findings showed that residents with high education and low income consumed the least alcohol daily. $10.4 \%$ of females in rural areas consumed alcohol. 80-90\% of married women aged 25 or older belonged to majority-drinking groups.

These statistics on alcohol consumption might affect the health or the onset of health problems and fitness of the Chinese women. Alcohol drinking patterns in China today are different from the historical folklore where alcohol consumption was part of a daily diet. For the daily diet, people often consumed alcohol with a meal, only encouraged social drinking, and did not misuse alcohol. Zhang (2005) stated that there was a historical use of alcohol for the Chinese as part of rituals, educational arts, poems and social events. The impetus for this present research study into the motivation, expectations and insights of Chinese women's alcohol consumption is fueled by the above health concerns. 


\section{Application of Expectancy Theory to Chinese Women's Alcohol Use}

Previous research studies showed that the changing roles of women and increased female involvement in society's workforce have influenced women's drinking behavior, as has the way women are portrayed in alcohol advertisements (Hao et al., 2004; Hao et al., 2005; Hao \& Young, 2000; Women and Alcohol, 2008). Learning from the drinking patterns in today's China, one might wonder what the motivational expectations behind Chinese women's alcohol consumption are. Alcohol expectancy indicates a contingent representation between a drinking behavior and its outcome; and has a significant effect on individual's cognition of alcohol consumption (Edwards et al., 1973; Smith \& Goldman, 1994; Bandura, 2004; Newman et al., 2006; Shell et al., 2009; 2010). This knowledge will illustrate the reasons why individuals decide to drink obsessively or in moderation. Therefore, understanding alcohol expectancy is essential for investigating why Chinese women believe their drinking behaviors are appropriate and for predicting their future drinking habits.

\section{Women Alcohol Expectancy}

Expectancy theory refers to a motivational contingency representation between a behavior and its outcome. An expectancy correlates to a behavior, predicts onset of a behavior and mediates a behavior and its outcome. Applying the concept of expectancy, women who drink may suffer depression as a result of being widowed or separated and feel the need for jiu (alcohol or wine) in order to release aversive emotions. Women who drink may be influenced by social activities (family festivals and ceremonies), and feel the need for jiu in order to experience joy or excitement. Chinese women who drink may 
be affected by the feudal ethical codes political system, and need jiu for the expression of freedom (Xu, 2003; Shu, 2004; He, 2004; Wang, 2008 and Huo, 2001).

Chinese feudal ethical roles of the Confucian political system referred to males as superior to females in society. Male's norms were to be wise, righteous, father, husband, and guides. Female norms were to obey father, husband and sons; guide the family order and marry only once. Women needed to be faithful to their family, possess physical charm, present polite speech and be good at needlework, such as quilting, managing house chores, nurturing the family and children, and respecting elders and her husband (Huo, 2001; Lin, 2001; Wang, 2008). The following reviews are empirical studies related to women's alcohol expectancy in China and the west.

\section{Psychological Needs}

There are quite a few studies indicating the importance of psychological needs to women's alcohol consumption. Women's alcohol consumption behaviors might spring from psychological needs, such as the need to suppress negative emotions or excite positive emotion or for sexual enhancement. Situations which might lead to alcohol consumption include couple separation, becoming a widower, being alone, or being with others who are drinking. These examples illustrate the burdens or joyful expressions of women who use alcohol. Women seem to cope with stressful life problems with nonaggressive actions (Cooper et al., 1992).

Kim et al. (2009) examined the level of alcohol consumption among Chinese college students in Hong Kong. 411 sophomore students from one of four colleges in Hong Kong in 2006 and 2,630 freshmen from the Chinese University of Hong Kong in 2005 participated in the study. The instruments were a self-administered assessment of 
demographic information, smoking status, drinking history, history of past-month binge drinking, symptoms of alcohol abuse and how often and how much individuals consumed alcohol. If students consumed five or more servings of alcohol per session, they were qualified as a binge drinker or alcohol dependent. Results found that binge drinking or alcohol misuse in college was associated with celebration (joyful experiences) and depression (stressful experiences) among female students.

Some studies, like Mondaini, N., Cai, T., Gontero, P., Gravazzi, A., Lombardi, G., Boddi, V., and Bartoletti, R. (2009), Davis et al. (2010) and Gradham \& Massak (2007), suggested that alcohol expectancy had an impact on women's alcohol consumption and the risk of sexual activities. The following assessments related expectations of sex to alcohol use among women. Davis et al. (2010) examined the influences of alcohol expectancy on women drinkers' sexual decision-making process. 94 college women who engaged in underage drinking participated in the study. Women with inhibiting cognition only had $\mathrm{r}^{2}=0.23$ positive association with discouraging sexual behavior, but had a negative $r^{2}=0.29$ association with encouraging sexual behavior. Both impelling and inhibiting cognition associated negatively with unprotected sexual intention with $\mathrm{r}^{2}=0.51$. Women's unprotected sexual behavior positively correlated with impelling cognition $\left(\mathrm{r}^{2}\right.$ $=0.61)$. These findings pointed out that underage women's drinking behaviors were indirectly mediated by their sexual cognitive intentions, increasing unprotected sexual behavior. At least one alcohol expectancy dealt with sexual activity outcomes, and women's sexually inhibiting cognitions did not prevent them from engaging in unprotected sexual behaviors. Women's psychological needs (i.e. sex, negative mood, 
positive mood) might be seen as a motivator (expectancy) that influences their drinking behaviors.

\section{Social Needs}

Women's drinking behaviors might spring from social needs, such as the need for a social or personal relationship. Women danced and drank with poets at the banquets during the Tang and Song dynasties (He, 2004; Shu, 2004). Women chose to drink at family reunions, birthday parties, funerals, weddings, and during the Chinese New Year (Hao et al., 1995; Hao and Young, 2000). The assessments through qualitative studies on alcohol use indicated this aspect of how social needs affect women's alcohol consumption.

Li, X., Fang, X., Stanton, B., and Feigelman, S. (1996) examined the relationship between alcohol use and students' social activities, problem behaviors and demographic status. 1,040 students in $6^{\text {th }}, 7^{\text {th }}, 8^{\text {th }}$ and $10^{\text {th }}$ grades from five public schools in Beijing participated in the study. The instrument that they used was a self-administered questionnaire. Their finding was drinking behaviors were positively associated with participation in social activities such as dating, parties, and family reunions. The following year, a study done by Lu et al. (1997) found a similar result.

In the west, two qualitative studies also indicated the same pattern that young women viewed alcohol consumption as pleasurable and fun, a part of normal social life. Women who misused alcohol viewed binge-drinking behaviors as a pattern from masculine gender identity, and one that was different from traditional female roles (Rudolfsdottir and Morgan, 2009; Lyons and Willott, 2008). Lyons and Willott (2008) explored how alcohol consumption of young women in New Zealand was associated with 
female social positions and gender identities. Their findings were that young women viewed alcohol consumption as delightful or entertaining, a part of normal social life. The setting of alcohol use seemed to be empowered social interaction among young women, providing enjoyment and a feeling of security among friends.

Need for Freedom

Women's alcohol consumption behavior might spring from gender freedom or equality. For example, modern writers called for political freedom, and equality between men and women in Chinese society (He, 2004; Shu, 2004; Li, 2010). The dramatic socioeconomic changes in China and western influences might leverage women's expectations of alcohol consumption as well (Hao et al., 1995; Hao and Young 2000). Other research studies in the western and eastern countries have supported this trend of women's alcohol expectancies.

Chinese women's alcohol expectancies might be influenced by their role experiences in today's China, and the definition of that role has been argued (Hao et al., 1995; Lin, 2001). Lin (2001) addressed issues of females' roles in China from the old days to the modern days. The traditional role of a female in the society and culture of feudal China was to obey her husband, and to be inferior. Today, Chinese women get an education and are involved in societal activities as men are. Lin made the extraordinary point that in China, today's women are trapped at a crossroads on gender issues and women's value in society. For instance, female writer Zhang Xin lost her husband's love due to her feminist perspectives. Modern female writers in China sway between Western feminist trends and female feudal ethical roles. Parallel results to Lin's study were also found in Shell et al. (2009); Wang (2008) and Wong (2000). 
In the west, other discussion of social trends and interpersonal factors indicated a relationship between alcohol consumption and gender roles. Women's drinking patterns in Australia changed somewhat due to the high economic growth (Roche, 2001). For example, drinking by women changed from being unacceptable to socially supported. The traditional role for a woman is the family's nurturer, but now the drinking level of single women between 18-23 years old (without children) and women in the labor force is the same as the males' drinking level in Australia. Roche et al. suggested that women's role in society had changed for reasons such as labor force participation and higher education, and that now women are unlikely to become mothers until their 30s.

A five-year longitudinal follow-up study on gender roles and drinking patterns among Czech Republic women by Kubicka and Csemy (2008) addressed how women's roles in modern society influenced their drinking behavior as well. Gender role orientation data was collected in 1992 from a sample of 468 women in Prague, and their drinking patterns were observed for five years. They found that egalitarian and hedonistic gender role orientations (non-traditional gender roles) revealed motivation for women's alcohol consumption. Egalitarian (freedom) women consumed $\geq 90 \mathrm{~g}$ alcohol per session. Women with a hedonistic (pleasure seeking) gender role orientation consumed $\geq 48 \mathrm{~g}$ per session or $\geq 40 \mathrm{~g}$ of alcohol daily. These two studies seemed to show that societal patterns for women did tie into the development of alcohol consumption expectancy among women.

In conclusion, the perceptions surrounding drinking behaviors of women in China and the west, and their expectations of alcohol consumption revealed three categories (psychological needs, social needs and the need for freedom) into which drinking 
behaviors may fall. The rationale for developing a measurement scale for Chinese women's alcohol expectancies through a mixed method study was inspired by the studies done by Smith \& Goldman (1994) and Shell et al. (2009; 2010).

\section{Men and Women's Alcohol Expectancy}

In today's China, the societal norms for men and women show that women might have social, psychological, and egalitarian expectations that can be related to alcohol consumption. Historically, researchers such as Bongers et al. and Shell et al. have looked for differences between men's and women's alcohol expectancy. Both researchers' findings have shown no significant differences between men's and women's alcohol expectancy, but that men scored higher on alcohol positive expectancy and lower on negative expectancy than women (Edwards et al., 1973; Smith and Goldman, 1994; Bongers et al., 1998; Shell et al., 2009; 2010). However, there still is a need to review studies in the west and China on alcohol expectancy related to both men and women. These studies provide direction for the present study, which may help determine if alcohol expectancy significantly differs by gender.

\section{Men's and Women's Alcohol Expectancy in the West}

In the west, some research studies have shown that men's and women's alcohol expectancies were different. Coopers et al (1992) found that women coped with alcohol related problem situations, like stress, through active styles of emotion coping. However, male drinkers with avoidant styles of coping with stress (anger-in or anger-out) strongly associated with emotion-related effects of alcohol use and misuse. Rauch and Bryant (2000) found that gender differences in alcohol expectancy (sexual enhancement and 
tension reduction) were significant. Women had greater expectations for sexual enhancement and alcohol use than men. Men had greater tension reduction from alcohol use than women.

Lyons et al. (2006) examined alcohol expectancy between female and male drinkers. Their findings indicated that alcohol consumption perspectives in social and media messages were different between men and women. Men's alcohol expectancy involved war and battle metaphors; women's alcohol outcome expectancy aligned with working hard, pleasurable activities and relaxation. This qualitative study examined text from magazines that portrayed alcohol consumption for both men and women.

Similar results were also found in another qualitative study done by Lyons and Willott (2008). Moreover, Graham and Massak (2007) found a relationship between depression and heavy alcohol consumption among women, but not men. Rudolfsdottir and Morgan (2009) conducted a qualitative study that explored the alcohol consumption perspectives of middle class women and determined that alcohol consumption was different in men and women, and women learned to develop their own meaningful drinking expectancy themes. In addition, women tended to be influenced by environmental factors more than men, for example, in a social context where people tend to drink more. However, if alcohol use and its effects are placed in a lone, or personal, context people tend to drink less. This effect has been named the 'companion effect' (Maisto et al., 1999, p. 117).

Overall, a great deal of research has been conducted on defining alcohol expectancy for men and women. Women appeared to consume alcohol to relieve unpleasant emotions, to seek intimacy in relationships and to look more sophisticated. 
For men, alcohol consumption was associated only with the relief of unpleasant emotions. This research only involved qualitative studies, and not measurement approaches. This issue leads to the question of whether there is a better approach that allows us to measure significant differences between men's and women's alcohol expectancy.

\section{Men's and Women's Alcohol Expectancy in China}

Alcohol consumption by Chinese women has been on the rise, but Chinese women seem to be concerned because of the rise in alcohol consumption with the view of traditional female roles, or loss of dignity in front of friends and family members. The assumption from Hao and Young's (2000) study was that Chinese women's alcohol expectancy might be influenced by traditional feudal roles. This idea was also consistent with previous studies in the west, which indicated that women's alcohol expectancy is affected by social factors. Few empirical studies have been conducted in China about men's and women's alcohol expectancy. There are two mixed methods studies related to Chinese men's and women's alcohol expectancy.

Shell et al. (2009) looked for differences in alcohol expectancy among male and female Chinese students who were various types of drinkers (nondrinkers, occasional drinkers and regular drinkers). 1,244 high school students from two cities in Inner Mongolia completed the CAEQ (Chinese Alcohol Expectancy Questionnaire, by Newman et al. 2006). Findings indicated there were no significant differences in alcohol expectancy between females and males, although males scored higher on positive expectancy and lower on negative expectancy than females did. Male students generally had higher alcohol-related positive expectancy than female students. Shell et al. (2010) 
examined the associations among alcohol expectancy, self-efficacy, cultural orientation, and drinking behaviors. 1,020 students (512 male, 506 female) from three Beijing high schools completed Newman et al.'s (2006) CAEQ (Chinese Alcohol Expectancy Questionnaire). They found that drinkers held more positive than negative alcohol expectancies, and the frequency of drinking was increased among drinkers with more western beliefs than those with traditional Chinese beliefs. Also, men and women had no significant differences in negative expectancy. The study's findings appeared similar to those of Shell et al. (2009), and indicated that the gap in negative alcohol expectancy between Chinese men and women has narrowed since 2009.

In summary, reviewing the literature on Chinese women's alcohol expectancy, and men's and women's alcohol expectancy, has showed that alcohol expectancy is predictive of drinking behavior and it motivates an individual's behavior. Women might seek traditions, psychological pleasure and socialization through alcohol consumption, while men might seek power as well as pleasure and socialization. In summary, while the suggestions researchers provided in their studies explained the reasons why alcohol consumption has increased among Chinese women in today's society, evidence which illustrates the innate meaning of the motivating factors behind women's drinking behaviors is not clear. No empirical studies exist from the perspective of Chinese women regarding the consumption of alcohol in China.

Newman et al. (2006); Shell et al. (2009); and Shell et al. (2010) provided the only discussion related to Chinese expectations of alcohol consumption in China. The findings from these studies signified there was no significant difference on alcohol expectancy by gender. A new qualitative approach that asks both men and women about 
Chinese women's expectations for alcohol consumption, however, could yield more information on alcohol expectancies and gender differences. Thus, the present study applies expectancy theory to develop and validate a Chinese women's alcohol expectancy instrument through a mixed method study, and examines whether there is a significant difference between men's and women's alcohol expectancies. 


\section{CHAPTER THREE}

Study 1 was a qualitative methods study. The purpose of study 1 was to collect a wide range of qualitative data to better understand Chinese college-age people's attitudes, perceptions and knowledge about alcohol expectancies. This work was to guide and build the groundwork for developing items for inclusion in the scales that I planned to validate in study 2, based on people's experience, public perception of the role of Chinese women's alcohol drinking expectancies, and motivational perceptions of Chinese women who consume or do not consume alcohol. In study 1, I was to interview people (males and females) randomly in various places in China. The study was approved by the University of Nebraska - Lincoln, Institution Review Board (see Appendix A). The following details steps taken to develop a female alcohol consumption expectancy scale through qualitative methods.

\section{Method}

\section{Participants}

One hundred and thirty-four (74 male and 60 female) interviewees in this study. Interviewees were divided into two groups by age range: the first group of people (ages 18-25+), had 58 male and 54 female subjects; and the second group (ages 40-50+), had 16 males and 6 females. 50 males and 46 females (ages 18-25+) and 6 males and 5 females (ages 40-50+) participated in interview groups. In one-on-one interviews, the number of males and females in the 18-25+ age group were 8 and 8 respectively; and from the 40-50+ age group, 11 males and 1 female. All participants in the study were chosen by random chance from different public locations like bars, shops and restaurants. 


\section{Materials}

For the pilot study, I worked with experts in the field brainstorming questions related to alcohol consumption to ask local Chinese immigrants in Lincoln before conducting actual interviews. Based on the pilot study results (see Appendix B), I finalized a set of semi-structured interview questions.

The four semi-structured interview questions were asked during interviews. During the random group discussions and one-on-one interviews, and in participationobservation groups, the four key questions were followed to keep interviewees' statements consistently pertaining to the topic.

\section{Semi Structured Interview Questions}

The key questions used for interviewees were the following:

1) What do women expect through alcohol drinking?

2) What do men think about women who drink?

3) What do you (interviewee's self) think of women who drink alcohol?

4) What do other people think of women who drink alcohol?

All interview sessions were recorded using a digital recorder (SONY R IC recorder ICD-UX200). Recordings made in the bars or nightclubs, restaurants and streets captured background noises that were made while interviewees participated in drinking games, and at dinner or breakfast tables. The data were loaded into a Windows laptop. A notebook and a Kodak Easy Share C330 digital camera were used for antenatal records. 


\section{Procedure}

\section{Interview place selections}

All interviews were done as I found chance opportunities in wide range communities, in Kaiping, Guang Zhou and Hong Kong, China in June and July, 2010. For the random group interviews, also by chance, I came across groups sitting at a dinner table or drinking table with an average group size of six people in various places (bars, restaurants, street bars and shops).

The main interviewing and participation happened in the region of Kaiping, Guang Dong, China. Kaiping city has a population of 680,000 and is located 140 kilometers away from Canton (Guang Zhou) city, the capital of Guang Dong province, in southern China. Cantonese and Kaiping dialects are the official languages in the city. I interviewed 120 Kaiping residents in many settings closely related to Chinese women's consumption of alcohol. These settings are bars, nightclubs, snack shops, clinics, beauty salons, street bars, fashion shops, foot massage centers and breakfast restaurants. Additionally, five secondary or follow-up interviews were conducted after relationships were formed with interviewees in bars and restaurants. The establishment of a social environment helped the researcher connect with interviewees.

Second, I interviewed 10 Guang Zhou residents. Guang Zhou is the capital city of the Guang Dong province, with a population of $10,334,500$. It is located in southern China on the Pearl River and is about 120 kilometers northwest of Hong Kong. The official languages are Cantonese and Mandarin. The interviews happened in the following areas: taxi and bus stops, restaurants, an alcohol shop, and in neighborhood 
housing in Che Guang, Canton. The interviewees were taxi drivers, shop customers, and gatekeepers. The last community was the city of Hong Kong, an 1,104 $\mathrm{km}^{2}$ area located on China's south coast and surrounded by the South China Sea with a population of 7.03 million. The official languages are Cantonese and English. I led a random discussion group of four Hong Kong residents. Two of them (a female and a male) had worked in a bar for eight years. This interview happened in a bar.

Interview Transcribing Steps

I listened to each interview video and wrote down key words in both English and Cantonese. I used the blind research technique of not separating men's and women's responses, in order to identify patterns about women's expectations of alcohol consumption without bias. Each interview segment was listened to a second time while journal entry notes were compiled. Then notes from the interviews and the journal were summarized. Following this, I teamed with two experts in the field and searched the information for trends in women's drinking behaviors. 


\section{CHAPTER FOUR}

\section{Results}

Over a 2-month period in China, I collected preliminary data on the perceptions surrounding alcohol consumption behaviors of women and found six patterns in alcohol expectancies. These six patterns are five psychological themes, health themes, social themes, family themes, economic themes and the need for freedom from gender roles. These trends seemed to fall into the three categories of Chinese women's alcohol consumption expectations found in the literature (psychological needs, social needs and the need for freedom from gender roles). Five psychological themes and three health themes belong to the psychological needs category. The social needs category holds four social themes, and family and economic themes. The need for freedom from gender roles is a psychological self-expression theme. The five psychological themes in the psychological needs category are relief of anxiety, redefining one's image, selfexpression, sharing one's perspective, and positive feelings. The four social themes are meeting lovers, expanding friend circles, exploring nightlife entertainment, and having experiences among friends of friends. Family needs display one theme, which is maintaining family traditions, and economic needs also have one theme, which is business, or professional drinking. Finally, a health needs category has three themes: good appearance, remedy and negative thoughts.

\section{Discussion}

The qualitative data from Study 1 was used to develop and to assess the Chinese women's alcohol expectancy instrument in study 2 . The work revealed six prominent categories into which these drinking behaviors may fall: 1) social needs; 2) psychological 
needs; 3), family needs; 4) economic needs; 5) health needs; and 6) need for freedom from gender roles.

First, women's drinking behaviors may spring from their social needs, such as the need or hope for a social or personal relationship. Jiu is seen as a means of facilitating exciting social experiences, for instance, one-night stands with people met in bars and clubs for wealthy women, or meeting new friends / girlfriends / boyfriends in entertainment nightclubs. Females who want to go out to drink feel the need to meet wealthy friends in order to gain access to increased social entertainments and opportunities. Alternatively, married women may feel that their responsibilities at home with children, or at work, are preventing them from connecting with society. (See social needs theme 1 and theme 4: i.e. "If I drink, then I will explore more entertainment options," or "If I drink, then it will help me fulfill my sexual needs."). A few examples inform the social needs as follows (see transcribed examples in Appendix F):

Interviewer: What do you think of women who drink alcohol?

Lady 3: Women drinking ads, but use women to sell alcohol products a lot.

Man 1: normally on TV women drink alcohol

Lady1: like going out with friends at bars or MTV

Man 2: like hanging out with friends in bars or KTV outside. It means that you have to drink beers; otherwise, you will not be happy after drinking.

Lady 2: No...no, only use women to sell alcohol ads because women wear less, and attract more people to buy beers, plus, those women are very sexy.

Man A: women have many purposes to drink alcohol

Man B: for example, some women who drink because of they like the taste of alcohol, but some women like to Pie jiu

Interviewer note: Pie jiu is a kind of entertainment, which means to drink, drank, drunk.

Second, women's alcohol consumption behaviors may spring from psychological needs for an emotional and complicated excitement. One example was, "If I drink, I will be able to forget the sadness of a broken relationship," (see psychological needs theme 1). Interviewees phrased this idea to say that a married woman's relationship (bad or good) with her husband will affect whether she chooses to drink alone or with others, to either 
forget a burden or express joy. Another example from interviewees was that some women enjoy drinking with men and showing passion in love. Some women don't need a reason to drink, but some women need to drink to express feelings of loneliness (see psychological needs, themes 3 and 4). For college age women facing failure, struggles, or success at school, at home or at the work place, drinking helps them to relax (See psychological needs theme 2: "If I drink, I will be more competitive, thoughtful, or be able to ignore reality and just be happy with other people"). The following interview excerpts illustrate the psychological needs (see transcribed examples in Appendix $F$ ):

Interviewer: What do women expect through alcohol drinking?

Man A: A group of women will not order beers while chatting, but would have red wine while chatting. Women drink wine from happiness. If a person drinks happily at the table, you will drink with a passion. There is no pressure. For example, if you are sad, the heart is very uncomfortable with a sad feeling...nervous... after drinking, you can release your emotion and become open to speak up whatever you want to say.

Man B: It is about women who look for men to have intimate relationships with. Women will look for men in bars to fulfill other kinds of needs (i.e. sex).

Man A: reduce pressure...need attractive feelings in society...

Thirdly, drinking behaviors of Chinese women also spring from the family needs for job celebrations, unions, birthday parties, funerals, weddings, festivals, and anniversaries. In each occasion, women's drinking purpose may focus on various brands of jiu. Some women preferred red wine while drinking with friends, and chose Blue Belt or Baiwei beers for drinking competition games in bars with friends (see family needs theme 1: "If I drink, it will help me respect and socialize better with family and friends at reunions"). An example demonstrates the family needs as follows (see transcribed examples in Appendix $F)$ :

Interviewer: What do you think of women who drink alcohol? Man A: women choose to drink western wine. Man B: you can add some juice to mix so that you can drink sweetly Man A: women choose to drink red wine, expect they drink beers for competition in bars with men. 
Man B: sometime, it depends on drinking passions in season, women choose to drink Blue Belt, Baiwei and Li Jia. We sometimes need to have celebration parties, drinking will connect with celebrations at school.

Fourth, women who drink alcohol might look for economic benefits from business drinking at the dinner table or other places of entertainment (see economic needs theme 1). Traditional family and business drinking are one part of the Chinese drinking cultures (Wong, 2000; Wei et al., 2005). The following quotes from the interview excerpt reflect the theme of business drinking (see transcribed examples in Appendix F).

Interviewer: What do you think about women who drink alcohol?

Male: $\quad$ More women drink in today's China.

Interviewer: why? Would you explain to me a little bit?

Male: $\quad$ Economics developed in society, human being living condition gets better in China...so not like women who stayed at home in the old days, women could work and play. Interviewer: what do you mean by work and play?

Male: $\quad$ in the old days, only men work outside, and only one income for one family. Men needed to feed family...now people get wealthy and they could think about alternative life joyful activities such as entertainments - drinking alcohol...nowadays, men could earn money, so do women; men could drink, women have the right to drink too...

Interviewer: What do you mean by drinking alcohol and business?

\section{Comments from a male and female}

In many regions in China, people prefer solving problems at the dinner table. Drinking is a dinner table problem. Last month, there was a customer, a Shandong woman, who said that knowing how to drink is like understanding how to conduct business. Without drinking, nothing can happen in a business career. She shared her experience with us; she would first drink 12 cups of jiu at the dinner table and then could have a chance to talk about business. According to her sharing, we can tell drinking seemed to be a force for making a living in society under many circumstances.

A fifth need is health. Women's alcohol consumption behaviors might spring from the need for beauty or longevity, to feel sexy, to be in the current popular, and to appear youthful (see health needs theme 1: "choosing brands and quality of Jiu matters to women's drinking"). For example, some women suggested drinking red wine each day to prevent cancer. Some women believed drinking red wine might help them become healthy and get nutrition from alcohol for the kidney, stomach and liver, and to gain longevity (see Appendix F, “酒 jiu provides you a healthy benefit”). Elderly women 
prefer to drink a little jiu each day, to aid their blood flow. Former researchers also found this trend in their studies (Newman et al., 2006; Wong, 2008; Shell et al., 2006; 2010).

Sixth, women drink alcohol from the need to be free, intelligent. Women choose to drink differently based on individual thoughts on freedom. The following was an interview sample (see transcribed examples in Appendix F):

Interviewer: What do you think about women who drink alcohol?

Female: The reasons of women who drink alcohol were women wanted to show men their power and freedom.

For example, women drink nothing for a particular partner or over-drink without getting drunk without reason. Another example is, "groups of females call for political freedom, equality between men and women in the society," (see psychological needs theme 2: "If I drink, then I will feel superior to other people or my self-efficacy will increase").

In summary, this qualitative groundwork guided me to the creation of items to include in the expectancies instrument. A quote mentioned by several interviewees during one-on-one interviews reflects these themes. The statements were phrased as follows:

Jiu (alcohol) is a kind of drink, but mixed with human feelings [based on the feeling, Jiu becomes a different type of drink and allows human beings to experience something else]. Life is like a cup of jiu, mix into a big crowd [a group of different people drinking together], and experiencing one another's feelings, thus, life becomes sour, sweet, bitter, and spicy. If you are happy, cheer up [drink jiu] with some friends; if you are sad, alcohol can give you a good night's sleep; if you want to express yourself, drinking alcohol can give you an excuse.

The fact that more and more women drink alcohol in China relates to the complexity of society and human feelings and thoughts. Chinese women seem to be motivated to drink alcohol under the influences of these needs, psychological needs, social needs and desires for freedom, which are drinking behavioral outcomes. These findings were supported by the literature. These patterns seem to reflect the motivational 
perspectives that Chinese women have about consuming or abstaining from alcohol in China. 


\section{CHAPTER FIVE}

Study 2 is a quantitative methods study. The purpose of the study was to provide initial development and validation of the items that I established and to determine that these items constitute a legitimate psychometric instrument that reflects Chinese women's alcohol expectancies. The study was done to gain a better understanding of Chinese women's expectations of alcohol consumption, and if they were significantly different from men's alcohol expectancies. In study 2, I first developed the alcohol expectancy instrument based on the preliminary data of themes from study 1 ; then I established 82 separately unique items in these themes or categories for conceptual clarification (see Table 1). Finally, as the instrument was established, validation of the items and an examination of whether men and women respond significantly differently on Chinese women's alcohol expectancy instrument were conducted through exploratory factor analysis and independent t-tests. Study 2 was approved by the University of Nebraska - Lincoln, Institution Review Board (see Appendix A).

\section{Method}

\section{Items Development}

Six patterns were identified in study 1: 1) social needs; 2) psychological needs; 3), family needs; 4) economic needs; 5) health needs; and 6) need for freedom from gender role (see Table 1). Those trends seemed to fall in the three categories of Chinese women's expectations of alcohol consumption in the literature. 300 statements were drafted, which reflected trends in Chinese women's drinking behaviors. Selected examples are in Appendix $C$. I then selected six graduate students in the field, asked for 
feedback on the statements, and eliminated redundant statements. The second draft included 200 statements. These 200 statements were originally transcribed into the Cantonese and Kaiping dialects.

Second, I teamed with a group of four sophomore Chinese students at a large midwestern U.S. university. Each student translated the 200 statements into Mandarin Chinese. Then, I combined the two draft translation segments, and also considered the translation given by Google's online service. Further redundant statements were eliminated from the second draft. The third draft contained 148 statements. The fourth draft of the statements added an expectancy scale with personal statements and contained 96 items. Two experts and six graduate Chinese students in the field each provided comments on the 96 expectancy statements (i.e. if and then relationship - see Table 1 and Appendix D), and following this feedback I converted the 96 alcohol expectancy statements to be neutral for both genders (male and female). For example, a statement specific to women, "If I drink, I will be able to forget the sadness of a broken relationship with my husband," was changed to a gender neutral statement, "If I drink, I will be able to forget the sadness of a broken relationship with my partner." The purpose was to reinvestigate whether there were significant alcohol expectancies differences between men and women through the quantitative methods study. Further redundancy was edited, and the expectancy scale statements were written in the form, "If I drink, I will or it will."

Three Chinese and American students and two experts in the field evaluated the 96 statements for possible redundancy within the context of Chinese social and alcohol expectancy. A pre-test was performed within a group of 25 students in mainland China. The purpose was to check the accuracy of the Chinese translation, and if the instrument 
was legible students. A draft measurement of Chinese women's alcohol consumption

expectancy was finalized, with 82 Chinese women's expectancy statements written in Standard English and Chinese Mandarin in study 1 (see Appendix E).

\section{Table 1}

Preliminary Data: Themes observed in the drinking behaviors of Chinese women Psychological Needs:

Theme 1: relief of anxiety and emotional behaviors

1. If I drink, then it will help me release my anger.

2. If I drink, then it will help me overcome anxiety.

3. If I drink, I will be able to forget the sadness of a broken relationship.

4. If I drink, then it will help me release emotions from relationships with work mates.

5. If I drink, it will relieve my stress.

6. If I drink, it will relieve my boredom.

7. If I drink, it will help me define or identify who I am.

8. If I drink, then I will be more relaxed.

\section{Theme 2: redefining one's image or values}

1. If I drink, then I will feel respected.

2. If I drink, then I will feel attractive.

3. If I drink, then I will feel like and independent person.

4. If I drink, I will feel good about myself.

5. If I drink, then I will feel superior to other people.

6. If I drink, then I will feel more mature.

7. If I drink, then my self-efficacy will increase.

8. If I drink, then I will be a more successful business person.

9. If I drink, then I will feel I am a gentle and elegant person.

10. If I drink, then I will be a more open and talkative person.

11. If I drink, then I will be a more playful and fun person.

12. If I drink, then I will be a more responsible partner.

13. If I drink, it helps me to be a better lover.

14. If I drink, then I will be livelier in social drinking occasions.

15. If I drink, then I will be more honest.

16. If I drink, I will be able to enjoy life's mysteries without having to solve them.

17. If I drink, then I will be more creative.

18. If I drink, I will feel I am a dreamer.

19. If I drink, then I will be warmer towards other people.

20. If I drink, then I will be more enthusiastic about helping other people. 
21. If I drink, then I will feel sexy.

22. If I drink, then I will have a "bad girl" or "bad boy" image.

23. If I drink, then I will be more competitive.

24. If I drink, then I will be more thoughtful.

25. If I drink, then I will not have to justify myself to other people.

\section{Theme 3: self-expression}

1. If I drink, then I will be more confident in front of other people.

2. If I drink, it will become my drinking habit.

3. If I drink, then I will be able to ignore reality and just be happy with other people.

\section{Theme 4: sharing others' perspectives (perspective taking)}

1. If I drink, then I will be able to confidently talk with someone whom I have nothing in common with.

2. If I drink, then I will be allowed to cry without being judged.

3. If I drink, then I will be able to discuss my life with friends.

4. If I drink, then it will help me see other perspectives and better communicate with other people.

5. If I drink, then it will let others know that I am a strong person.

\section{Theme 5: positive feelings}

1. If I drink, then I will have a quiet moment in which to be myself.

2. If I drink, I will have a moment of drinking enjoyment.

3. If I drink, I will have an excuse to be in love.

4. If I drink, then I will get a good night's sleep.

5. If I drink, then I will be able to imagine living in a fantasy world.

6. If I drink, then I will be able to share my thoughts with other people, friends, or family.

7. If I drink, I will be able to stand up for those who go against tradition.

\section{Social Needs/Sexual needs:}

\section{Theme 1: meet lovers}

1. If I drink, then it will help me fulfill my sexual needs.

2. If I drink, it will help me to fulfill my exploration of drinking in which observing other people's drinking behaviors.

3. If I drink, then my sexual performance will improve.

\section{Theme 2: expand circle of friends (girlfriends or boyfriends)}

1. If I drink, then I will be able to understand and accept other people more easily.

2. If I drink, then I will explore more entertainment options.

3. If I drink, then I will develop supportive friendships outside of school.

4. If I drink, then I will feel like I can understand other people' feelings.

\section{Theme 3: explore entertainments with friends or strangers}

1. If I drink, then I will be able to meet wealthy people.

2. If I drink, then I will be able to explore the social nightlife. 
3. If I drink, it will help me communicate with others about coping with work and family problems.

4. If I drink, then I will be able to have fun with people at night after a day of hard work.

\section{Theme 4: learning experiences among friends of friends}

1. If I drink, I will be able to explore different kinds of drinking experiences

2. If I drink, then I will understand how society functions.

3. If I drink, then I will be more in tune with popular culture or be caught up on pop culture and world news.

4. If I drink, it will help me pursue gender equality.

5. If I drink, then I will be able to share my stories freely with others in public.

6. If I drink, then I will be able to look for friends who care about my feelings.

7. If I drink, it will help me exchange worldviews or cultural perspectives within groups of different.

8. If I drink, it will help me reconnect with society after being married.

\section{Family needs:}

\section{Theme 1: maintain friends/family traditions}

1. If I drink, I will have more passion to celebrate holidays and special events.

2. If I drink, it will help me respect and socialize better with family and friends at reunions.

3. If I drink, I will be able to get reacquainted with old friends.

\section{Economic needs:}

\section{Theme 1: business professional drinking}

1. If I drink, then it will help me overcome barriers in the business world.

2. If I drink, then I will be showing respect to other people who toast me at the dinner table.

3. If I drink, then I will have self-control while drinking during business dinners.

\section{Health needs}

\section{Theme 1: good looking due to social influences}

1. If I drink, it will help me to appear better looking and popular.

\section{Theme 2: remedy}

1. If I drink, it will help to prevent cancer.

2. If I drink, I will get a nutritional benefit from the alcohol.

3. If I drink, it will increase my blood flow).

4. If I drink, I will be at less risk for heart disease.

\section{Theme 3: negative thoughts}

1. If I drink, then I will feel dizzy

2. I drink, then I will feel more irritated.

3. If I drink, then I will lose my temper.

4. If I drink, then I will feel hopeless about my future. 


\section{Participants}

One thousand five hundred and fifty participants, undergraduate students randomly selected from thirteen universities in three provinces, Beijing, Yunnan and Wuhan, China and who were not part of study 1, participated in the survey. 401 college students (male: 181 , female: $201 ; 19$ missing values; Mean $=20.47$, and $S D=1.728$ ) in Beijing Foresy University completed the survey. 516 college students (male: 216, female: 297; 4 missing values; Mean $=20.26$, and $S D=1.311$ ) in three Yunnan universities: Kunming Medical University, Yunnan University and University of Finance and Economics; completed the survey. 633 college students (male: 306, female: 326; 2 missing value; age Mean $=20.75$, and $S D=3.43$ ) from eight universities in Wuhan provinces completed the survey. The study was conducted in schools. All participants completed the Chinese women's alcohol expectancy questionnaire with a pencil and an eraser.

\section{Materials}

To examine the Chinese women's alcohol expectancy instrument, I selected experts in the field and recruited students in selected classes who were told about the survey and its purpose. Whole classes were selected and all students in the classes were invited to complete the questionnaire. The 82-item Chinese women's alcohol expectancy instrument was used in study 2. Participants' responses were measured on a 5-point Likert scale ( 1 =strongly disagree, $2=$ disagree, $3=$ neutral, $4=$ agree, and 5=strongly agree) . In addition, there were four demographic variables (age, gender, grade and hometown) for each participant in the scale. This instrument was administered as part of a larger set of instruments. 


\section{Procedure}

\section{Survey Place Selections}

Study 2 involved Beijing Normal University, the Yunnan Health Education Institute, and the Chinese Center for Health Education, Ministry of Health (MOH). Participants were in a wide range of communities; the study 2 data was collected at universities in Beijing, Wuhan, and Kunming, China.

The following details steps taken to examine the instrument. All participants from the three areas' universities answered the questionnaire in a regular classroom with a trained data collector supervising in May, 2011. Participants listened to a verbal description of the project, which also was provided to them in a written form, and then answered the questions in the questionnaire. Participants completed this survey within 30 - 35 minutes. Completed questionnaires were gathered by data collectors and taken from the school immediately. Trained investigators from the three cooperating institutes in China gathered data from students in intact classes just as the administration would collect an academic test or exam. The computerized data set was compiled by data entry professionals in China.

Data Analysis

The computerized data files were sent back to the University of Nebraska Lincoln, Educational Psychology Department. I performed data analysis using the SPSS 19 statistical package to identify latent variables. 25 participants did not complete some items $(\geq 10)$ and 10 participants did not fill out " 1 " for male or " 2 " for female under the gender demographic variable in the survey, so a total of 35 cases were deleted. The gender demographic variable is the one that I was using for further data analysis, and 
also, the second fold of the study was to define whether there were gender differences on alcohol expectancy. A total of 1515 cases were used for final data analysis (male: 698, female: 817 ; age range 17-30, Mean $=20.48$ and $S D=1.696$ ). Item analysis via Exploratory Factor Analysis (EFA) and independent $t$-test were conducted in study 2 . 


\section{CHAPTER SIX}

\section{Results}

Findings in study 2 indicated the Chinese women's alcohol expectancy instrument was reliable and valid statistically and conceptually with $\alpha>0.7$ (see Table 3, 4, and 5). The hypothesis was met in study 2; there were significant differences between males and females on alcohol expectancy across 7 latent variables.

\section{Objective 1: Validate Chinese women's alcohol expectancy instrument}

First, EFA was conducted with principal components extraction and Varimax rotation, using a minimum factor loading $\geq 0.3$ as a cut off score for items of each factoring score. At the first round of EFA, I chose a 12-factor solution (Eigenvalues $\leq$ 0.1), and the findings showed items and factor loadings were low, and the cluster items were not matched with the themes in study 1. Expectancy items were combined based on multiple loadings in the 12-factor solution. Then, a 10-factor solution was applied (Eigenvalues $\geq 0.3$ ). Items and the factor loading scores were low for two sets of cluster items (i.e. factor loading scores < 0.6) and the meanings for cluster items did not conceptually represent the themes either.

The decision was made to choose Eigenvalues greater than or equal to 0.3. An 8factor solution was applied. Items and the factor loadings were low at three factors' highest score (i.e. factor loading scores $<0.6$ ), and there were six sets of cluster items matching the meaning of themes that had been identified in study 1 . Therefore, along with two experts in the field, I decided to exclude 21 items from the 8 -factor solution. The 8-factor solution, its items and factor loadings for two factors' highest score were 
still low (i.e. factor loading scores $<0.6$ ), then a 7-factor solution was conducted. The items and factor loadings in the 7-factor EFA solution were consistent (i.e. factor loading scores $>0.6$ ). The meaning of each factor corresponded to the original themes in study 1 reasonably well. I also performed a 6- factor and a 5-factor solution. Findings showed that the items and factor loadings in each solution were inconsistent (had missing values for more than two factors), and there was no conceptual consistency with the themes. The next step was to compare the 8-factor and 7-factor solutions, and decide which one was the best solution.

In the second round of EFA, I discussed both the 7-factor and 8-factor solutions with two experts and two Chinese graduate students in the field, focusing on thematic meanings and items, and factor loadings. Two factors seemed to recollapse on the same theme in the 8-factor solution, however, the 7-factor solution better separated the themes, and reflected conceptual and thematic fit. Finally, nine items were deleted from the data and the 7-factor solution was chosen. The items were examined for conceptual consistency with other items related to the themes. Based on these criteria, the seven factors were identified as final latent variables of the instrument; and a total of 30 items that were conceptually and statistically poorly fitted were eliminated. A reliability test was followed up for each factor (Cronbachs' alpha > 0.7).

Reliability is a necessary pre-condition for validity. The seven identified factors (sub-scales) matched the themes (see Table 2). The 7-factor solution for the instrument had the best psychometric and conceptual fit. The themes of Chinese women's drinking behaviors provided a conceptual meaningfulness of each sub-scale loading in EFA. These factor names were conceptually established as follows. 
Factor one refers to a self-confidence dimension, or self-images/values. It reflects the study 1 freedom thoughts theme, that women who drink might seek intelligence and choose to drink differently based on individual negative or positive emotions.

Factor two refers to social perception, in which drinking enhances social functioning and communication, and helps women keep up with their social network. This factor matched the themes of family traditions, experiences with friends and business drinking under social needs, economic needs and family needs in study 1. Social, hope or personal relationships also fall under the social perspective.

Factor three refers to thoughts of freedom from gender roles, and the excitement of positive mood. Psychological needs represent an emotional excitement and joyful expression. This factor matched four themes under psychological needs in study 1 .

Factor four portrays negative mood reduction, or illustrates suppression of negative emotional behaviors. This factor relates to the self-anxiety theme under psychological needs. Women described the need to drink a lot of alcohol because of their loneliness, or failure or success, at school and work. Drinking helps them to release emotion or anger.

Factor five refers to sexual enhancement or enhancement of intimate relationships. It mirrors the first social needs theme, to meet lovers or explore entertainment; from study 1, women enjoy drinking with men and showing passion to gain love opportunities.

Factor six is conceptualized as a representation of traditional home health remedies, such as the need for beauty and longevity. 
Factor seven refers to personal negative expectancy, and results in mental, physical and social consequences. These ideas reflect the third health needs theme: the negative personal thoughts from study 1 . Women might not drink alcohol because they consider that their reputation may be ruined through alcohol consumption; or they feel uncomfortable after drinking alcohol.

Table 2

Similarities and Differences between Themes in Study 1 and 7-factors in Study 2 Similarities (Factors Paired with 1 Theme)

Study 1 - Themes Psychological Needs

Redefining one's image or values

Relief of anxiety/emotional behaviors

Sexual Needs

Meeting lovers

Health Needs

Remedy

Personal Negative thoughts

\section{Study 2 - 7-Factors}

Self-Confidence

Negative Moods

Intimacy Relationship

Health

Negative Personal Expectancy

\section{Differences (Factors paired with 3 or more Themes)}

\section{Psychological Needs}

Self-expression

Sharing other's perspectives

Positive feelings

Redefine one's values and images

Social needs/Family Needs/Economic Needs

Family traditions

Business drinking

Experiences with friends
Freedom Thoughts (positive mood)

Notes: Themes in italics refer to a category of needs about women's alcohol consumption. Under each category are women's alcohol expectancy themes. 
Seven latent variables in the final scales were named based on the themes. Cronbach's alpha values for each factor were as follows: self-confidence $=0.92$; social perception $=0.89 ;$ freedom thoughts $=0.86$; negative $\operatorname{mood}=0.83 ;$ intimacy relationship $=0.82$; health $=0.79$; and personal negative expectancy $=0.71$. These items each have item-total correlations greater than 0.6 (Cronbach's alpha $=0.7$ is considered to show internal consistency of the measurement), and item-total correlations range from 0.39 to .80 , which indicate that these items are good measures of the seven identified dimensions of the Chinese women's alcohol expectancy study (see Table 3).

Second, a conceptually meaningful 7-factor solution was obtained by conducting a principal-component analysis with Varimax rotation separately for male and female groups, and was to ensure that the factor structure defining the scales was common across groups. The goal was to verify the latent variables (7-factor) were adequate (reliable and valid) for male and female alcohol expectancies on the scale separately. In the first round data analysis for males' and females' alcohol expectancies, the result showed items and factor loadings for the male group were inconsistent from those of the female group by two poor items: one item under factor four in the male group had no value, and the other item was not loaded in the same factor that was shown in the female group. These two unfitted items were excluded because of inconclusive factor loadings and conceptual inconsistency. The final scale had 50 items.

In the second round of data analysis for both genders, the items and factor loadings in the male group were consistent with those of the female group in the final instrument (see Table 4 and 5). Final results revealed that factors corresponded to male and female alcohol expectancies consistently. Items and alpha score by genders (male 
and female) were the following: self-confidence, $\alpha=0.92$ and $\alpha=0.92$. Social perception, $\alpha=0.88$ and $\alpha=0.88$. Freedom thoughts, $\alpha=0.86$ and $\alpha=0.86$. Negative mood, $\alpha=0.82$ and $\alpha=0.83$. Intimacy relationship, $\alpha=0.80$ and $\alpha=0.83$. Health, $\alpha=0.76$ and $\alpha=0.81$. Negative personal expectancy, $\alpha=0.67$ and $\alpha=0.75$; male and female respectively. All the items and factor loadings across 7-latent variables in men and women's scales were verified the same, which showed that the instrument was reliable and valid. The remaining 50 items in the final solution were used to measure gender differences on Chinese women's alcohol expectancy.

\section{Table 3}

Chinese Women's Alcohol Expectancy Items and Factor loading for the Final Instrument Items and Scales

\begin{tabular}{llllllll}
\hline Item & F1 & F2 & F3 & F4 & F5 & F6 & F7 \\
\hline
\end{tabular}

\section{Factor 1: Self-confidence $(\alpha=.92$, item $n=11)$}

13. If I drink, then I will feel superior to other people. $\quad .77$

16. If I drink, then I will be a more successful business person.

10. If I drink, then I will feel attractive. $\quad .77$

14. If I drink, then I will feel more mature. $\quad .72$

15. If I drink, then my self-efficacy will increase. $\quad .72$

9. If I drink, then I will feel respected. $\quad .72$

17. If I drink, then I will feel I am a gentle $\quad .70$ and elegant person.

11. If I drink, then I will feel like $\quad .68$ and independent person.

21. If I drink, it helps me to be a better lover. $\quad .62$

20. If I drink, then I will be a more responsible partner. $\quad .58$

19. If I drink, then I will be a more playful and fun person. .57 
Factor 2: Social perception $(\alpha=.89$, item $n=9)$

72. If I drink, then I will be showing respect to other people $\quad .79$ who toast me at the dinner table.

73. If I drink, then I will have self-control $\quad .74$ while drinking during business dinners.

70. If I drink, I will be able to get reacquainted with old friends. $\quad .73$

68. If I drink, I will have more passion to celebrate holidays and $\quad .72$ special events.

69. If I drink, it will help me respect and social better .68 with family and friends at reunions.

71. If I drink, then it will help me to overcome the barriers .68 in the business world.

62. If I drink, then I will be more in tune with popular culture $\quad .53$ or be caught up on pop culture and world news.

65. If I drink, then I will be able to look for friends who care about my feelings.

66. If I drink, then it will help me exchange worldviews or cultural perspectives with people from different groups.

\section{Factor 3: Freedom thoughts $(\alpha=.86$, item $n=8)$}

25. If I drink, then I will be more creative.

32. If I drink, then I will be more thoughtful.

26. If I drink, I will feel I am a dreamer.

24. If I drink, I will be able to enjoy life's mysteries without having to solve them.

23. If I drink, then I will be more honest.

42. If I drink, then I will have a quiet moment in which to be myself.

28. If I drink, then I will be more enthusiastic about helping other people.

43. If I drink, I will have a moment of drinking enjoyment.

79 68 68

Factor 4: Negative $\operatorname{mood}(\alpha=.83$, item $n=7)$

2. If I drink, then it will help me overcome anxiety.

5. If I drink, it will relieve my stress.

4. If I drink, then it will help me to release emotions $\quad .71$

from relationships with work mates.

$\begin{array}{ll}\text { 1. If I drink, then it will help me to release my anger. } & .69 \\ \text { 3. If I drink, I will forget my sadness of a broken relationship. } & .65\end{array}$

$\begin{array}{lll}\text { 3. If I drink, I will forget my sadness of a broken relationship. } & .65 \\ \text { 8. If I drink, then I will be more relaxed. } & .35 & .46\end{array}$

6. If I drink, I will relieve my boredom.

Factor 5: Intimacy Relationship ( $\alpha=.82$, item $n=6)$

57. If I drink, then I will be able to explore 32 the social nightlife.

56. If I drink, then I will be able to meet wealthy people. $\quad .37$

53. If I drink, then I will explore more entertainment options.

54. If I drink, then I will develop supportive friendships outside of school.

51. If I drink, then my sexual performance will improve.

44. If I drink, then I will have an excuse to be in love.

\section{Factor 6: Health $(\alpha=.79$, item $n=4)$}

76. If I drink, I will get a nutritional benefit.

78. If I drink, I will be at less risk for heart disease.

75. If I drink, it will help me to prevent cancer.

77. If I drink, it will increase my blood flow. 


\section{Factor 7: Personal negative expectancy}

$(\alpha=.71$, item $\mathrm{n}=5)$

80. I drink, then I will feel more irritated.

81 . If I drink, then I will lose my temper.

82. If I drink, then I will feel hopeless about my future.

Notes: Items are shown in the order obtained in the final exploratory factoring. Total participants, $\mathrm{n}=1515$. F1 $=$ Factor 1 and so on. All factors shown on the table loaded at least $\geq 0.3$. Items that had highest loadings together are shown as a factor. Cronbach's alpha was calculated as reliability statistics of the instrument $(\alpha=0.7)$, and are shown in parentheses following each factor's name.

\section{Table 4}

\section{Chinese Women's Alcohol Expectancy Items and Factor loading for Male Final Instrument Items and Scales}

\begin{tabular}{llllllll} 
Item & F1 & F2 & F3 & F4 & F5 & F6 & F7 \\
\hline
\end{tabular}

\section{Factor 1: Self-confidence $(\alpha=.92)$}

16. If I drink, then I will be a more successful. $\quad .77$ business person.

10. If I drink, then I will feel attractive. $\quad .74$

13. If I drink, then I will feel superior to other people. $\quad .73$

14. If I drink, then I will feel more mature. $\quad .72$

15. If I drink, then my self-efficacy will increase. $\quad .68$

9. If I drink, then I will feel respected.

17. If I drink, then I will feel I am a gentle $\quad .66$ and elegant person.

11. If I drink, then I will feel like and 64 independent person.

21. If I drink, it helps me to be a better lover. $\quad .62 \quad .31$

20. If I drink, then I will be a more responsible partner. $\quad .60 \quad .39$

19. If I drink, then I will be a more playful and fun person. $.51 \quad .46$

\section{Factor 2: Social perception $(\alpha=.88)$}

72. If I drink, then I will be showing respect to other people $\quad .79$ who toast me at the dinner table.

73. If I drink, then I will have self-control

trinking during business dinners.

70. If I drink, I will be able to get reacquainted with old friends. $\quad .70$

68. If I drink, I will have more passion to celebrate holidays and $\quad .70$ special events.

71. If I drink, then it will help me to overcome the barriers $\quad .68$ in the business world.

69. If I drink, it will help me respect and social better .64 with family and friends at reunions.

62. If I drink, then I will be more in tune with popular culture $\quad .55$ or be caught up on pop culture and world news.

65. If I drink, then I will be able to look for friends .52 who care about my feelings.

66. If I drink, then it will help me exchange worldviews or cultural perspectives with people from different groups. 


\section{Factor 3: Freedom thoughts $(\alpha=.86)$}

25. If I drink, then I will be more creative.

26. If I drink, I will feel I am a dreamer.

23. If I drink, then I will be more honest.

24. If I drink, I will be able to enjoy life's mysteries

.61

without having to solve them.

32. If I drink, then I will be more thoughtful.

28. If I drink, then I will be more enthusiastic about helping other people.

42. If I drink, then I will have a quiet moment in which to be myself.

43. If I drink, I will have a moment of drinking enjoyment.

$\begin{array}{lll}.31 & .32 & .51\end{array}$

$.31 \quad .44$ .35

$.41 \quad .36$

Factor 4: Negative $\operatorname{mood}(\alpha=.82)$

2. If I drink, then it will help me overcome anxiety.

1. If I drink, then it will help me to release my anger.

5. If I drink, it will relieve my stress.

4. If I drink, then it will help me to release emotions from relationships with work mates.

3. If I drink, I will forget my sadness of a broken relationship.

8. If I drink, then I will be more relaxed.

6. If I drink, I will relieve my boredom.

\section{Factor 5: Intimacy Relationship $(\alpha=.80)$}

57. If I drink, then I will be able to explore the social nightlife.

56. If I drink, then I will be able to meet wealthy people. $\quad .35$

53. If I drink, then I will explore more entertainment options.

44. If I drink, then I will have an excuse to be in love.

51. If I drink, then my sexual performance will improve.

54. If I drink, then I will develop supportive friendships

outside of school.

\section{Factor 6: Health $(\alpha=.76)$}

76. If I drink, I will get a nutritional benefit.

78. If I drink, I will be at less risk for heart disease.

75. If I drink, it will help me to prevent cancer.

\section{Factor 7: Personal negative expectancy $(\alpha=.67)$}

80. I drink, then I will feel more irritated.

81. If I drink, then I will lose my temper.

82. If I drink, then I will feel hopeless about my future.

79. If I drink, then I will feel dizzy.

30. If I drink, then I will have a "bad boy" or "bad girl" image.

Notes: Items are shown in the order obtained in final exploratory factoring for male participants $(\mathrm{n}=698)$. $\mathrm{F} 1=$ factor 1 and so on. All factors shown on the table loaded at least $\geq 0.3$. Items that had highest loadings together are shown as a factor. Cronbach's alpha was calculated as reliability statistics of the instrument $(\alpha=0.7)$, and are shown in parentheses following each factor's name. 
Table 5

Chinese Women's Alcohol Expectancy Items and Factor loading for Female Final Instrument Items and Scales

$\begin{array}{llllllll}\text { Item } & \text { F1 } & \text { F2 } & \text { F3 } & \text { F4 } & \text { F5 } & \text { F6 } & \text { F7 }\end{array}$

Factor 1: Self-confidence $(\alpha=.92)$

13. If I drink, then I will feel superior to other people. $\quad .80$

10. If I drink, then I will feel attractive. $\quad .78$

16. If I drink, then I will be a more successful business person. $\quad .76$

9. If I drink, then I will feel respected. $\quad .74$

15. If I drink, then my self-efficacy will increase. $\quad .73$

17. If I drink, then I will feel I am a gentle and elegant person. $\quad .72$

14. If I drink, then I will feel more mature. $\quad .71$

11. If I drink, then I will feel like and independent person. .70

19. If I drink, then I will be a more playful and fun person. .60

21. If I drink, it helps me to be a better lover. $\quad .60$

20. If I drink, then I will be a more responsible partner. $\quad .52$

Factor 2: Social perception $(\alpha=.89)$

72. If I drink, then I will be showing respect to other people $\quad .79$ who toast me at the dinner table.

73. If I drink, then I will have self-control $\quad .75$ while drinking during business dinners.

70. If I drink, I will be able to get reacquainted with old friends. $\quad .74$

68. If I drink, I will have more passion to celebrate holidays and $\quad .74$ special events.

69. If I drink, it will help me respect and social better $\quad .71$ with family and friends at reunions.

71. If I drink, then it will help me to overcome the barriers $\quad .67$ in the business world.

62. If I drink, then I will be more in tune with popular culture $\quad .51$ or be caught up on pop culture and world news.

65. If I drink, then I will be able to look for friends $\quad .50$ who care about my feelings.

66. If I drink, then it will help me exchange worldviews $\quad .48$ or cultural perspectives with people from different groups.

Factor 3: Freedom thoughts $(\alpha=.86)$

25. If I drink, then I will be more creative.

32. If I drink, then I will be more thoughtful.

24. If I drink, I will be able to enjoy life's mysteries without having to solve them.

26. If I drink, I will feel I am a dreamer.

42. If I drink, then I will have a quiet moment in which to be myself.

23. If I drink, then I will be more honest. 74 67 51

28. If I drink, then I will be more enthusiastic about helping other people.

$\begin{array}{lll}\text { 43. If I drink, I will have a moment of drinking enjoyment. } & .30 & .48\end{array}$

.64

.62

.60

.60

.55

.54

.53 
Factor 4: Negative mood $(\alpha=.83)$

2. If I drink, then it will help me overcome anxiety. $\quad .76$

4. If I drink, then it will help me to release emotions $\quad .75$

from relationships with work mates.
5. If I drink, it will relieve my stress.

1. If I drink, then it will help me to release my anger.

3. If I drink, I will forget my sadness of a broken relationship. $\quad .65$

8. If I drink, then I will be more relaxed.

6. If I drink, I will relieve my boredom. $\quad .42$

\section{Factor 5: Intimacy Relationship $(\alpha=.83)$}

57. If I drink, then I will be able to explore the social nightlife..32

56. If I drink, then I will be able to meet wealthy people. $\quad .34 \quad .64$

53. If I drink, then I will explore more entertainment options. $\quad .36 \quad .61$

54. If I drink, then I will develop supportive friendships $\quad .35 \quad .60$ outside of school.

51. If I drink, then my sexual performance will improve.

44. If I drink, then I will have an excuse to be in love.

Factor 6: Health $(\alpha=.81)$

76. If I drink, I will get a nutritional benefit.

78. If I drink, I will be at less risk for heart disease. $\quad .82$

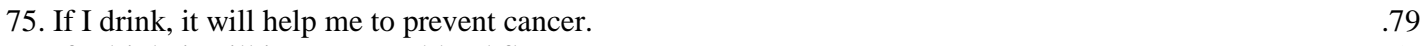

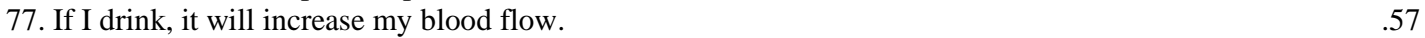

Factor 7: Personal negative expectancy $(\alpha=.75)$

80. I drink, then I will feel more irritated. $\quad .81$

81. If I drink, then I will lose my temper. $\quad .75$

82. If I drink, then I will feel hopeless about my future. $\quad .75$

79. If I drink, then I will feel dizzy. $\quad .31 \quad .63$

30. If I drink, then I will have a "bad boy" or "bad girl” image. $\quad .30 \quad 49$

Notes: Items are shown in the order obtained in the final exploratory factoring for female participants $(\mathrm{n}=817)$. $\mathrm{F} 1=$ factor 1 and so on. All factors shown on the table loaded at least $\geq 0.3$. Items that had highest loadings together are shown as a factor. Cronbach's alpha was calculated as reliability statistics of the instrument $(\alpha=0.7)$, and are shown in parentheses following each factor's name.

\section{Objective 2: Examine alcohol expectancy differences between Chinese men and women.}

I performed an independent $t$-test; effect size was computed following the test.

The goal was to examine if there were significant differences in alcohol expectancies by

genders. Table 4 and Table 5 show the similar alpha scores of each factor between male

and female groups for self-confidence, social perception, freedom thoughts and negative

mood; the factors with different alpha scores by genders are intimacy relationship, health 
and negative personal expectancy. For items and factor loadings, males had higher positive perceptions than females; but females had higher intimacy relationship perception than males.

Mean scores and $\boldsymbol{t}$-test results for gender comparisons shown in Table 6 indicate that women's alcohol expectancy significantly differed from men's in each factoring scale with a p-value less than 0.001 ; except one factor under health with test statistic pvalue less than 0.05 . Effect size was calculated for each latent variable. The t statistic in each factor was as follows: Factor one (self-confidence), $\mathrm{t}(1513)=8.868, \mathrm{p}<0.001, \mathrm{~d}=$ .45. Factor two (social perception), $\mathrm{t}(1513)=4.062, \mathrm{p}<0.001, \mathrm{~d}=.21$. Factor three (freedom thoughts), $\mathrm{t}(1513)=5.253, \mathrm{p}<0.001, \mathrm{~d}=.27$. Factor four (negative mood), $\mathrm{t}(1513)=4.008, \mathrm{p}<0.001, \mathrm{~d}=.21$. Factor five (intimacy relationship), $\mathrm{t}(1513)=7.480, \mathrm{p}$ $<0.001, \mathrm{~d}=.38$. Factor six (health), $\mathrm{t}(1513)=2.300, \mathrm{p}<0.05, \mathrm{~d}=.12$. Factor seven (personal negative expectancy), $\mathrm{t}(1513)=-3.633, \mathrm{p}<0.001, \mathrm{~d}=.19$. 
Table 6

Students' Mean Scores on Chinese Women's Alcohol Expectancy by Gender

\begin{tabular}{|c|c|c|c|c|c|c|}
\hline \multirow{2}{*}{ Factors } & \multicolumn{2}{|c|}{ Male } & \multicolumn{2}{|c|}{ Female } & \multirow{2}{*}{$\begin{array}{c}\text { Gender } \\
\text { Mean difference }\end{array}$} & Cohen's \\
\hline & $M$ & $S D$ & $M$ & $S D$ & & $d$ \\
\hline Self-Confidence & 2.30 & .746 & 1.98 & .675 & $.324 * * *$ & .45 \\
\hline Social-Perception & 3.31 & .761 & 3.15 & .751 & $.158 * * *$ & .21 \\
\hline Freedom Thoughts & 2.74 & .755 & 2.55 & .707 & $.197 * * *$ & .27 \\
\hline Negative Mood & 2.88 & .754 & 2.73 & .718 & $.152 * * *$ & .21 \\
\hline Intimacy Relationship & 2.65 & .740 & 2.36 & .722 & $.282 * * *$ & .38 \\
\hline Health & 2.51 & .776 & 2.42 & .781 & $.092 *$ & .12 \\
\hline Personal Ne Expectancy & y 2.76 & .708 & 2.89 & .718 & $-.134 * * *$ & .19 \\
\hline
\end{tabular}

Significant: $P<0.001 * * *, P<0.05^{*}$

$d=$ Effect size computed by Cohen's $d$; male, $\mathrm{n}=698$; female, $\mathrm{n}=817$.

Personal Ne Expectancy $=$ Personal Negative Expectancy

\section{Discussion}

One finding of study 2 showed that men's alcohol expectancies tended to be higher positives and lower negatives than women's alcohol expectancies. This is supported by previous studies (Shell et al., 2009; 2010; Newman et al., 2006; Bongers et al, 1998; Lyons and Willott, 2008; Smith and Goldman, 1994; Edwards, 1973). However, another finding in study 2 showed that females had higher intimacy perceptions than males on alcohol expectancy. This might be influenced by the cultural context, which indicates male drinking may be more acceptable to social expectations than female drinking. Another interpretation might be that women use active styles of emotional coping for dealing with alcohol use related to problematic situations like stress (unlike 
males). Women's alcohol expectancies were high in sexual enhancement as shown by the alignment with self-values, pleasurable activities and relaxation from negative emotions (Lyons et al., 2006; Graham and Massak, 2007; Bryant, 2000; Coopers et al., 2000; Peters et al, 2003; Davis et al., 2010).

The effect size of each factor showed that there was a meaningful significant difference on alcohol expectancies by genders. Compared to previous instruments on Chinese alcohol expectancy that were developed by Newman et al., (2006), Shell et al., (2009) and Shell et al., (2010), the current instrument showed there was a distinct separation between the two factors of thoughts of freedom from gender roles (positive mood) and negative mood for alcohol expectancies. These psychological needs and freedom thoughts of women's alcohol expectancies were reflected both in study 1 and study 2 .

Chinese women seemed to be motivated to drink by alcohol consumption outcomes that meet needs, such as psychological needs, self-confidence/values, social needs, intimate relationships with friends and others, gaining personal relationships, and thoughts or desires for freedom. Bolles' (1972) S-S* and R-S* model theory and Bandura's (1977) outcome expectancy help explain the meaning of an expectancy as an individual's belief about what outcomes are achievable through performing a designated behavior. These outcomes act as a motivator for individuals who intend on performing a behavior in order to achieve its outcomes. Thus, Chinese women expect these outcomes (motivational aspects) related to alcohol use, and these differ from those of males. I strongly suggest that future research repeats this methodological approach to studying gender differences on alcohol expectancies. In addition, knowing the expectations of 
women's alcohol consumption among Chinese women could help health professionals and educational psychologists make future predictions for women's drinking behaviors. 


\section{CHAPTER SEVEN}

\section{Overall Discussion}

The purpose of the present study was to develop the Chinese Women's Alcohol Expectancy Instrument (CWAEI), and to examine alcohol expectancy differences between males and females. Given the pioneering and cross-cultural nature of this study, a mixed method approach was used to explore alcohol expectancy among Chinese women. Two months of interview work involved and explored alcohol expectancies of Chinese women from the perspectives of both Chinese men and women. The groundwork led to the development of a final CWAEI 50-items. In this section, the major findings in studies 1 and 2 and their implications and limitations are discussed, along with suggestions for future research.

\section{Major Findings}

\section{Convergent findings}

Study 1

The CWAEI was formed to meet internal consistency requirements in the present study. Care was taken to assure that the CWAEI was tenable and conceptually sound with respect to the themes (validity). The themes that were identified in study 1 were supported by previous research in the west.

The theme of social needs divulges meeting lovers, expanding circles of friends and exploring nightlife entertainment. For example, Labrie et al. (2009) conducted a motivational-enhancement intervention group that had the objective of decreasing risky alcohol consumption behaviors. Their findings showed intervention group participants that significantly associated with high social motives appeared at lower risk of alcohol 
consumption behaviors weekly than participants in the control group with low social motives. Labrie et al. stated alcohol might have acted as a motivator for women who wanted to seek out new intimate relationships and social relations. Quantitative studies by Maisto et al. (1999) and Agrawal et al. (2007), also addressed the social motive themes, that females' lifetime alcohol consumption expectancies were attributed to environmental factors (i.e. social enhancement, family involvement in alcohol and peers). Davis et al. (2010) informed that expectation of a woman's impelling cognitions was positively associated with reasons to have sex through alcohol consumption. Social motives generally had a moderating effect on alcohol consumption.

The theme of psychological needs in study 1 , such as self-feelings, perspective taking, freedom thoughts, and self-performance were reflected in previous studies in the west. Findings from Cooper et al. (1992) indicated that females consumed alcohol related to problematic situations through active styles of emotion coping (i.e. neither anger-in nor anger-out). Kim et al. (2009), Goldstein (2006), Lyons et al. (2006) and Graham \& Massak (2007) found women's depression was associated with liquor and wine consumption, and doubts or celebration and relaxation about life situations. The relationship between alcohol consumption and depression showed among women, but not men.

The themes of alcohol expectancies on family, economy and health are family traditions, business professional drinking, remedy and negative thoughts. These themes also mimicked previous studies in the east and west. Examples were as follows: Hao et al. $(1995 ; 2000)$ indicated that the Chinese economy has grown rapidly, and business people believed drinking could enhance business meetings with friends at the dinner 
tables. Kubicka and Csemy's (2008) and Lyons and Willotts' (2008) studies pointed toward changes in women's roles that revealed motivation for consumption of alcohol. Women's daily red wine intake was beneficial for health and healthy sexual function (Lyons et al. 2006; Mondaini et al., 2009).

To summarize, Chinese women's alcohol expectancy themes in study 1 were supported by former research studies in the west and east. Study 1 found that Chinese women's alcohol expectancy relating to social and psychological needs were supported by western studies, and this might highlight the limited studies on Chinese women's motivational factors for alcohol consumption in the east. These findings seem to suggest that expectancies of social and psychological effects are more influential on women's motivation to drink.

Study 2

In study 2, the CWAEI was developed and examined for its reliability and validity through EFA; and it identified 7-factors using the sample of 1515 college students. Reliability analysis tested the internal consistency of 7-factors, and examined the results of CWAEI both conceptually and statistically. Finally, an EFA was applied to a men's and women's alcohol expectancy scale separately, and both scales were validated across 7-factors. Thus, the alcohol expectancy scales demonstrated an acceptable fit for men and women on alcohol expectancy. The final 50-items alcohol expectancy scales indicated an internally consistent measurement of the 7-subscales of alcohol expectancy. The research question in the present study was answered. Chinese women's alcohol expectancy reveals 7-factors in which is demonstrated women's alcohol consumption under social needs, the need of freedom from gender roles and psychological needs. These findings reflected 
those from previous studies in the west and east (Edwards, 1973; Smith and Goldman, 1994; Shell et al., 2009; 2010).

\section{Divergent Findings}

In the present study, 6-factors were similar to Brown et al.’s (1987) alcohol expectancy instrument, but one factor (health) was unique, although western studies have indicated a positive relationship between women's health and red wine consumption (Powers and Young, 2007; Mondaini et al., 2009). The health factor still reflected unique aspects of Chinese culture (i.e. if I drink, it will help me to prevent cancer; or if I drink, it will increase my blood flow).

The second divergent finding was that the intimate relationship factor on alcohol expectancy informed sexual enhancement (i.e. if I drink, then my sexual performance will improve) in the present instrument. Another divergent finding was that the freedom thoughts (positive moods) factor (i.e. if I drink, I will have a moment of drinking enjoyment) and negative moods (i.e. if I drink, I will relieve my boredom) are independent factors in CWAEI. These new findings were not presented in Shell et al.'s (2009; 2010) studies. This might demonstrate that Chinese women's alcohol expectancy derived from social and psychological needs.

\section{Gender}

A comparison of the CWAEI findings to those from Shell et al. (2009; 2010), and Smith and Goldman (1994) shows the major new finding in the present study to be the indication of significant differences in alcohol expectancies by genders. In study 2 , positive and negative alcohol expectancy was associated with alcohol consumption among males and females. Males have higher positive alcohol expectancies and lower 
negative than females, but items and alpha loading scores for females have higher alcohol expectancy for intimate relationships than males.

These findings suggested that males, who are more likely to drink, expect positive consequences to happen socially and physically. The findings were supported by previous studies (Donovan et al., 2009; Shell et al., 2009; 2010). Females have higher, "negative mood," "intimacy relationship," "health" and "negative personal expectancy" than men. The new finding seemed to indicate that women expect more sexual enhancement through alcohol use than men do. The motivational factors for women who consume alcohol seemed to be the relief of unpleasant emotions and to look sophisticated, while for men, alcohol consumption was associated only with the relief of unpleasant emotions. These criteria suggested women might be motivated to drink alcohol based on psychological needs, and health and freedom thoughts, and they differed significantly from males' motivations.

In summary, with the nature of the current instrument, the present study provides insights for future research and prevention practices in the following ways: it identifies Chinese women's alcohol expectancy - 7-factors. This statistical and descriptive profile of Chinese women's alcohol expectancies was supported by study 1 and study 2 , in addition to previous studies.

\section{Implications}

The significant contribution of the study was to clarify expectancy theory as an approach, which demonstrates the contingency representation between an expectancy and a behavior outcome. In this study, the results established a potential framework of understanding for Chinese women's expectations of alcohol consumption, and developed 
and validated a uni-dimensional instrument using mixed methods. I believe it is essential to consider Chinese women's customs, beliefs and practices when assessing alcohol expectancy and interpreting their meaning and significance.

Health professionals and educational psychologists in previous qualitative studies also argued there was a need to develop alcohol preventions in gender specific ways (Peters, et al., 2003; Roche, 2001; Rudolfsdottir and Morgan, 2009; Labrie, 2009; Smith and Goldman, 1994; Bandura, 2004; Stacy, 1990; 1997). The current instrument can be used for predicting future Chinese women's alcohol consumption behaviors; so that women's misuse of alcohol could be prevented. Alcohol prevention programs for women are limited. Only a few alcohol prevention programs exist in China at this time, and appear to be primarily focused on the alcoholic male. Alcoholics Anonymous, alcohol prevention programs in mental hospitals and alcohol prevention centers are focusing on male alcoholic patients only (San and Xun, 2000; Yu et al., 2006; Li, 2006). The current measurement scale provides information for future education on alcohol prevention, such as women's alcohol prevention or intervention in China.

The objective of developing the CWAEI was to pinpoint and to understand the reasons why women drink alcohol in China, relevant to the increasing concern regarding Chinese women's alcohol use. As the Chinese government pays less attention to alcohol consumption and regulation and more attention to economic growth, the question of how to apply the information from the present study to educational practices arises. Future research studies can apply expectancy theory to continue studying about women's alcohol expectancy worldwide, and gender differences on alcohol consumption behaviors through qualitative and quantitative mixed methods. Taking information from the scale, I 
expanded the views of the ways that Chinese women drink. For instance, Chinese women expect social enhancement through alcohol consumption. Health professionals or educational psychologists might want to think about developing community gatherings, or events such as board games at a potluck night. These events could offer alcoholic beverages. Through the community activities, women might meet their social needs through moderate alcohol use during social hours. Women and Alcohol (2011) suggested that middle-aged women appeared to have better health conditions through moderate alcohol consumption. It seems that moderate alcohol consumption is good for both women's health and psychological needs.

To extend the present study, expectancy theory can be adapted in educational programs for teachers, parents and students. Expectancy is a contingency presentation between a behavior and its outcome. For example, educational psychologists could use qualitative and quantitative mixed methods to create a psychometric measurement scale; a mathematical expectancy instrument pertaining to a target behavior. The behavior could be high school students' motivation to engage in studying math. In educational practice, educators could gain new perspectives on the reasons why high school students hate or love studying math in classrooms. There is no perfect predictor of future behavior, however, expectancy is still a better approach for explanations of motivational beliefs or goals that an individual values or disvalues, that lead the individual to choose or not choose behaviors (Stacy, 2006; Smith and Goldman, 1994).

\section{Limitations}

The findings from the present study will serve as a basis for future research on women's alcohol expectancy and gender differences. I believe there are a few limitations 
to this study. The first limitation is the large sample size, which skewed the validity of the instrument. This would affect the significance of the gender differences results. Second, there were no drinking behavior measurements to follow up the development and validation of the scales. Third, the present study does not address which expectancy factors of alcohol use might be significantly higher for men than women, or vice versa. Finally, the present study's applied samples were constrained to be in school environments, which limited generalization of these results to other populations (i.e. nonstudents or business drinkers) and settings in China. Thus, the sample used by this study might not be viewed as representative of China's nationwide population.

\section{Suggestions}

In spite of these limitations, I found that predicting future alcohol misuse among Chinese women was both difficult and sensitive. The advantage of the present study was that it is the first study to explore Chinese women's alcohol expectancy through mixed methods. This groundwork should be viewed as an exploration or development of expectancies' power to show the significant differences between males' and females' alcohol expectancy.

Future research studies will be able to address the generalization of the structure of alcohol expectancies, nationwide and internationally. Future researchers should clarify these results by repeating this study and examining whether there are legitimate significant differences on alcohol expectancy by genders. In addition, the application of expectancy theory to this alcohol expectancy instrument and drinking behavior measurement by gender should be followed up for future studies. Cranford et al. (2010) stated that positive outcome expectancies explained the reasons that individuals perform 
binge drinking. Drinking behavior expectancies measures could predict certain behaviors. An additional research question asks what differences might there be in terms of assessing both CWAEI and alcohol consumption measurement by genders.

It may also be interesting to study historical cultural values of Chinese women's alcohol consumption, and determine if this helps us gain more or different perspectives on the motivational expectations of Chinese women's alcohol consumption. He (2004) described Chinese women's wine culture in literature and outlined women's social and cultural background from dynasty to dynasty, as well as the alcohol consumption of educated women. In some legends, alcohol consumption by women in historical China has seemed to associate with depression. More than half the writings of Li Qing Zhao, a Song female poet, describe the loneliness and unhappiness she suffered during the country's famine and war, and illustrate the short-term emotional relief gained by drinking.

For the most part, I believe that studying historical values in human beings' complexity of cognitive thoughts on alcohol consumption could provide better conceptual support for the continuation of the validity of instrument development. Continuing the validation of the instrument will strengthen its reliability and validity. I strongly encourage other researchers in public health or educational fields to replicate the present study. The goal would be to continue investigating the distinct differences between men and women on alcohol expectancies in mainland China.

Overall, the present study provided scientific evidence of Chinese women's alcohol expectancies and developed the instrument for alcohol prevention programs. I invite educational psychologists and health professionals to continue investigating gender 
differences on alcohol expectancies. Knowing more about gender differences as a primary predictor of drinking (expectancies) will be of value in considering policy options to reduce alcohol-related risk and harm for men and women. 


\section{BIBLIOGRAPHY}

Agrawal, A., Dick, D. M., Bucholz, K. K., Madden, P. A. F., Cooper, M. L., Sher, K. J. and Heath, A. C. (2007). Drinking expectancies and motives: a genetic study of young adult women. Addition, 103, 194-204.

Ames, C. (1992). Classroom: goals, structures, and student motivation. Journal of Educational Psychology, 84(3), 261-271.

Bandura, A. (1977). Self-efficacy: Toward a unifying theory of behavioral change. Psychological Review, 84(2), 191-215.

Bandura, A. (2004). Health promotion by social cognitive means. Health Education \& Behavior, 31(2), 143-164.

Bartholow, B. D., Sher, K. J., and Strathman, A. (2010). Moderation of the expectancyalcohol use relation by private self-consciousness: data from a longitudinal study. Personality and Social Psychology Bulletin, 26(11), 1409-1420.

Benegal, V., Nayak, M., Murthy, P., Chandra, P., and Gururaj, G. (2005). Women and alcohol use in India. In Isidore, S. O., and Robin, R. (Eds.,). Alcohol, Gender and Drinking Problems (pp.90-123). World Health Organization.

Bolles, C.R. (1972). Reinforcement, expectancy and learning. Psychological Review, 79(5), 394-409.

Bongers, I. M. B., VanDeGoor, L. A. M., Van Oers, J. A. M., and Garretsen, H. F. L. (1998). Gender differences in alcohol-related problems: controlling for drinking behavior. Addiction, 93(3), 411-421.

Brown, S.A., Christiansen, B.A. and Goldman, M. S. (1987). The alcohol expectancy questionnaire: an instrument for the assessment of adolescent and adult alcohol expectancies. Journal of Studies on Alcohol, 48, 483-491.

Christiansen, B. A., Goldman, M. S., and Inn, A. (1982). Development of alcohol related expectancies in adolescents: separating pharmacological from social-learning influences. Journal of Consulting and Clinical Psychology, 50(3), 336-344.

Cochrane , J., Chen, H., Conigrave, K. M., and Hao, W. (2003). Alcohol use in China. Alcohol \& Alcoholism, 38(6), 537-542. 
Cooper, M. L., Russell, M., Skinner, J. B., Fromme, M. R., \& Mudar, P. (1992). Stress and alcohol use: Moderating effects of gender, coping and alcohol expectancies. Journal of Abnormal Psychology, 101 (1), 139-152.

Cranford, J. A., Zucker, R. A., Jester, J. M., Puttler, L. I., and Fizgerald, H. E. (2010). Parental alcohol involvement and adolescent alcohol expectancies predict alcohol involvement in male adolescents. Psychology of Addictive Behaviors, 24(3), 386396.

Davis, K. C., Norris, J., Hessler, D. M., Zawacki, T., Morrison, D. M. and George, W.H. (2010). College women's sexual decision making: cognitive mediation of alcohol expectancy effects. Journal of American College Health, 58(5), 481-489.

Donovan, J. E., Molina, B. S. G., and Kelly, T. M. (2009). Alcohol outcome expectancies as socially shared and socialized beliefs. Psychology of Addiction Behaviors, 23(2), 248-259.

Eccles, J. S. \& Wigfiled, A. (2002). Motivational beliefs, values, and goals. Annual Review of Psychology, 53, 109-132.

Edwards, G., Hensman, C. and Peto, J. (1973). A comparison of female and male motivation for drinking. International Journal of the Addictions, 8(4), 577-587.

Giang, K. B., Allebeck, P., Spak, F.,Minh, H. V. and Dzung, T. V. (2008). Alcohol consumption: Vietnam, alcohol use and alcohol consumption-related problems, in rural Vietnam: an epidemiological survey using AUDIT. Substance Use and Misuse, 43, 481-495.

Goldstein, B. I. (2006). Why do women get depressed and men get drunk? An examination of attributional style and coping style in response to negative life events among Canadian young adults. Sex Roles, 54(1/2), 27-37.

Graham, K. and Massak, A. (2007). Alcohol consumption and the use of antidepressants. CMAJ, 176(5), 633-637.

Hao, W., Young, D., Li, L., Xiao, S., Tu, J., Shen, H., Yu, Z., Teng, X., and Wan, X. (1995). Psychoactive substance use in three sites in China: gender differences and related factors. Addiction, 90, 1503-1515. 
Hao, W., Young, D., Xiao, S., and Li, L. (1999). Alcohol consumption and alcoholrelated problems: Chinese experience from six area samples, 1994. Addiction, 94(10), 1467-1476.

Hao, W., and Young, D. (2000). Drinking patterns and problems in China. Journal of Substance Use, 5(1), 71-78.

Hao, W., Su, Z., Liu, B., Zhang, K., Yang, H., Chen, S., Biao, M., and Cui, C. (2004). Drinking and drinking patterns and health status in the general population of five areas of China. Alcohol \& Alcoholism, 39(1), 43-52.

Hao, W., Chen, H., and Su, Z. (2005). China: alcohol today. Addiction, 100, 737-741.

Hays, R. (1985). An integrated value-expectancy theory of alcohol and other drug use. British Journal of Addiction, 80, 379-384.

He, J. (2004). Women poet and wine culture. China Academic, 11.[In Chinese]. http://www.cnki.net

Huo, J. (2001). Women of the Tang dynasty. China Today, 40-47.

Kim, J. H., Chan, K. W. C., Chow, J. K. W., Fung, K. P., Fong, Y. F., Cheuk, K. K. and Griffiths, S. M. (2009). University binge drinking patterns and changes in patterns of alcohol consumption among Chinese undergraduates in a Hong Kong University. Journal of American College Health, 58(3), 255-265.

Kubicka, L. and Csémy, L. (2008). Women's gender role orientation predicts their drinking patterns: a follow-up study of Czech women. Addition, 1-9.

Labrie, J. W., Huchting, K. K., Lac, A., Tawalbeh, S., Thompson, A. D., and Larimer, M. E. (2009). Preventing risky drinking in first-year college women: further validation of a female-specific motivational-enhancement group intervention. Journal of Studies On Alcohol and Drugs/Supplement, 16, 77-85.

Lambright, K. T. (2010). An update of a classic: applying expectancy theory to understand contracted provider motivation. Administration \& Society, 42(4), 375403.

Li, J., Ma, W., Xu, Y., et al. (2006). Investigation on drinking behaviors among inhabitants aged over 15 years in Guangdong province. China Journal of Public Health, 22(7), 775-776. 
$\mathrm{Li}, \mathrm{S}$. (2006). Conception and process of mental rehabilitation in alcoholic anonymous. Chinese Journal of Clinical Rehabilitation, 10(10), 161-164. [In Chinese]. http://www.zglckf.com

Li, S. (2010). Women poet and wine. The Art of Dining, 5-6. http://www.cnki.net

Li, X., Fang, X., Stanton, B., and Feigelman, S. (1996). The rate and pattern of alcohol consumption among Chinese adolescents. Journal of Adolescent Health, 19, 353361.

Lin, D. (2001). Chinese women's culture: from tradition to modernization. Chinese Education and Society, 33(6), 24-36.

Lu, Z. P., Engs, R. and Hanson, D. (1997) The drinking behaviors of a sample of university students in Nanning, Guangxi Province, People's Republic of China. Substance Use and Misuse, 32, 495-506.

Lyons, A. C., Dalton, S. I. and Hoy, A. (2006). Hardcore drinking portrayals of alcohol consumption in young women's and men's magazines. Journal of Health Psychology, 11(2), 223-232.

Lyons, A. C. and Willott, S. A. (2008). Alcohol consumption, gender identities and women's changing social positions. Sex Roles, 59, 694-712.

Ma, G., Zhu, D., Hu, X., Luan, D., Kong, L., and Yang, X. (2005). The drinking practice of people in China. Acta Nutrimenta Sinica, 27(5), 362-365.

Maisto, S. A., Carey, K. B., \& Bradizza, C. M. (1999). Social-learning theory. In Kenneth E. L. \& Howard, T. B. (2 ${ }^{\text {nd }}$ Eds.)., Psychological theories of drinking and alcoholism (pp.106-163). Guilford, NY: London.

Mendoza, M. R., Medina-Mora, M. E., Villatoro, J., and Durand, A. (2005). Alcohol consumption among Mexican women: implications in a syncretic culture. In Isidore, S. O., and Robin, R. (Eds.,). Alcohol, Gender and Drinking Problems (pp. 126-142). World Health Organization.

Mondaini, N., Cai, T., Gontero, P., Gavazzi, A., Lombardi, G., Boddi, V., and Bartoletti, R. (2009). Regular moderate intake of red wine is linked to a better women's sexual health. Journal of Sexual Medicine, 6, 2772-2777. 
Moss, A.C., and Albery, I. P. (2010). Are alcohol expectancies associations, propositions, or elephants? A reply to Wiers and Stacy (2010). Psychological Bulletin, 136(1), 17-20.

Newman, I. M., Qian, L., Shell, D. F., Qu, M., \& Zhang, Y. (2006). Development of Chinese adolescent alcohol expectancy questionnaire. Chinese Journal of Behavioral Medical Sciences, 15 (3), 274-276.

Peters, T. J., Millward, L. M. and Foster, J. (2003). Quality of life in alcohol misuse: comparison of men and women. Archives of Women's Mental Health, 6, 239-243.

Powers, J. R. and Young, A. F. (2007). Longitudinal analysis of alcohol consumption and health of middle-aged women in Australia. Addition, 103, 424-432.

Rauch, S. A. M., and Bryant, J. B. (2000). Gender and context differences in alcohol expectancies. The Journal of Social Psychology, 140(2), 240-253.

Redgrave, G. W., Swartz, K. L. and Romanoski, A. J. (2003). Alcohol misuse by women. International Review of Psychiatry, 15, 256-268.

Roche, A. M. (2001). Drinking behavior: A multifaceted and multiphasic phenomenon. In E. Houghton, \& A. M. Roche (Eds.), Learning about drinking (pp. 1-34). Philadelphia, PA: Brunner-Routledge.

Rudolfsdottir, A. G. and Morgan, P. (2009). Alcohol is my friend: young middle class women discuss their relationship with alcohol. Journal of Community and Applied Social Psychology, 19, 492-505.

Ryan, R. and Deci, E. L. (2000). Self-determination theory and the facilitation of intrinsic motivation, social development, and well-being. American Psychological Association, 55(1), 68-78.

San, L. and Xun, S. (2000). Alcohol misuse and treatment. Journal of Clinical Psychology and Medical Education, 10(6), 368-369. [In Chinese].

Schwarzer and Fuchs. (1995). Changing risk behaviors and adopting health behaviors: the role of self-efficacy beliefs. In Albert, B. (Eds.)., Self-Efficacy in Changing Societies. (pp.259-281). Cambridge University Press.

Shell, D. F., Newman, I. M., \& Qu, M. (2009). Alcohol expectancies among high school students in Inner Mongolia, China. Asia-Pacific Journal of Public Health, 21(4), 433-441. 
Shell, D. F., Newman, I. M., \& Fang, X. Y. (2010). The influence of cultural orientation, alcohol expectancies and self-efficacy on adolescent drinking behavior in Beijing. Addition, 105, 1608-1615.

Shu, H. (2004). Women literature and wine culture of the ancient China. Journal of Tangdu, 20(3), 80-83, [in Chinese].

Smith, G. T., and Goldman, M. S. (1994). Alcohol expectancy theory and the identification of high-risk adolescents. Journal of Research on Adolescence, 4(2), 229-247.

Stacy, A. W. (1997). Memory activation and expectancy as prospective predictors of alcohol and marijuana use. Journal of Abnormal Psychology, 106, 61-73.

Stacy, A. W., Widaman, K. F. \& Marlatt. (1990). Expectancy models of alcohol use. Journal of Personality and Social Psychology, 58 (5), 918-928.

Su, Z., Hao, W., and Chen, H. (2003). Alcohol patterns, alcohol consumption and alcohol-related problems in five areas in China. China Journal of Health Psychology, 17(8), 536-539.

Tang, Y. (1993). Alcohol anonymous theory and practices. Journal of Chinese Mental Health, 7(6), 265-267. [In Chinese]. http://www.cnki.net

Wang, A. (2008). Chinese family-development and changes. In Charles, H.B. and Stephan, M. (Eds.)., Families in a Global Context (pp.325-352). New York, NY, US: Routledge/Taylor \& Francis Group.

Wei, K., Deng, J., and Wang, Y. (2005). Alcohol consumption and patterns in Chengdu, Panzhihua. Journal of Panzhihua Science and Technology and Information, 27(3), $15-18$.

WHO (2004). Global Status Report on Alcohol. http://www.who.int/substance_abuse/publications/en/china.pdf

WHO. (2009). Alcohol. http://www.who.int/mediacentre/factsheets/fs349/en/

Wigfield, A., Tonks, S., and Eccles. J.S.(2004). Expectancy value theory in cross-cultural perspective. In Dennis, M.M. \& Shawn, V. E. (Eds.)., Big Theories Revisited (pp.3-18). Hillsdale, NJ: Erlbaum. 
Wilsnack, R.W., Wilsnack, S. C., and Obot, I. S. (2005). Why study gender, alcohol and culture? In Isidore, S. O., and Robin, R. (Eds.,). Alcohol, Gender and Drinking Problems (pp. 12-35). World Health Organization.

Women and Alcohol (2008). Institute of Alcohol Studies. http://www.ias.org.uk/resources/factsheets/women.pdf

Women and alcohol. (2011). Retrieved from http://www.icap.org/PolicyTools/ICAPBlueBook/BlueBookModules/9Womenand Alcohol/tabid/170/Default.aspx

Wong, S. P. (2000). Drinking banquet cultures in Tang dynasty. In Xu, S., and Chen, H. (Eds.,). Marching Toward the $21^{\text {st }}$ Century of International Alcoholic Beverage Culture - 2000 International Alcoholic Beverage Culture \& Technology Symposium. (pp.202-209). Journal of Northwest Institute of Light Industry.

Wu, B., Mao, Z.F., Rockett, I., \& Yue, Y.W. (2008). Socioeconomic Status and Alcohol Use among Urban and Rural Residents in China. Substance Abuse and Misuse, 43, 952-966.

Wu, Y. (2006). Reasons for women drink beer. Journal of Liquor Making, 33(1), 21-22. [In Chinese]. http://d.wanfangdata.com.cn/Periodical_nj200601011.aspx

Xiao, L. (n/a). Women and wine. LXQH, 29. China Academic Journal, [In Chinese]. http://www.cnki.net

Wu, L., D., S., and J. D. (2007). Alcohol misuse and treatment services among collegeage young adults. Psychiatric Service, 58(2), 192-200. [In Chinese].

Xing, Y., Ji, C., and Zhang, L. (2006). Relationship of binge drinking and other healthcompromising behaviors among urban adolescents in China. Journal of Adolescent Health, 39, 495-500.

$\mathrm{Xu}, \mathrm{F}$. (2003). Women and wine history. China and Drink, 46-47. [In Chinese]. http://www.chinadrinks.net

Yan, C. A. (2007). Influence of alcoholic dependence on the physical fitness state of university students. Journal of Clinical Rehabilitative Tissue Engineering Research, 11(30), 5968-5970. 
Yu, J., Jie, H., and Chang, Y. (2006). 59 cases of mental disorders caused by alcohol and alcohol treatments. Journal of Chinese Rural Medicine,13(1), 59-60. [In Chinese]. http://www.cnki.net

Zhang, J., Casswell, S., \& Cai, H. (2008). Increased drinking in a metropolitan city in China: a study of alcohol consumption patterns and changes. Addiction, 103(3), 416-423. 


\title{
APPENDICES
}

\section{Appendix A}

\section{Study 1 IRB Approved Form}

https://nugrant.unl.edu/irb/viewPrintedMessage.php?ID =51084

10/05/11 1:17 PM

\section{Nebrasika}

June 8, 2010

\author{
Ian Newman \\ Department of Educational Psychology \\ 232 TEAC, UNL, 68588-0345
}

IRB Number: 20100610932 EX

Project ID: 10932

Project Title: alcohol in chinese culture

Dear Ian:

This letter is to officially notify you of the approval of your project by the Institutional Review Board (IRB) for the Protection of Human Subjects. It is the Board $>$ s opinion that you have provided adequate safeguards for the rights and welfare of the participants in this study based on the information provided. Your proposal is in compliance with this institution Federal Wide Assurance 00002258 and the DHHS Regulations for the Protection of Human Subjects (45 CFR 46) and has been classified as exempt.

You are authorized to implement this study as of the Date of Final Approval: 06/08/2010. This approval is Valid Until: 12/01/2010.

1. Please contact Maria Moreno, Research Compliance Specialist, about Export Control Regulations related to your travel. Maria can be reached at 472-1837.

We wish to remind you that the principal investigator is responsible for reporting to this Board any of the following events within 48 hours of the event:

* Any serious event (including on-site and off-site adverse events, injuries, side effects, deaths, or other problems) which in the opinion of the local investigator was unanticipated, involved risk to subjects or others, and was possibly related to the research procedures;

* Any serious accidental or unintentional change to the IRB-approved protocol that involves risk or has the potential to recur;

* Any publication in the literature, safety monitoring report, interim result or other finding that indicates an unexpected change to the risk/benefit ratio of the research;

* Any breach in confidentiality or compromise in data privacy related to the subject or others; or

* Any complaint of a subject that indicates an unanticipated risk or that cannot be resolved by the research staff.

This project should be conducted in full accordance with all applicable sections of the IRB Guidelines and you should notify the IRB immediately of any proposed changes that may affect the exempt status of your research project. You should report any unanticipated problems involving risks to the participants or others to the Board. 
https://nugrant.unl.edu/irb/viewPrintedMessage.php?ID $=51084$

If you have any questions, please contact the IRB office at 472-6965.

Sincerely,

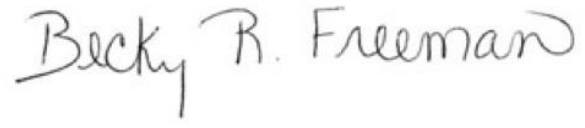

Becky R. Freeman, CIP

for the IRB

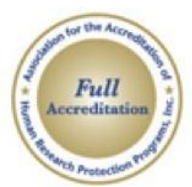

Page 2 of 2 


\section{Study 2 IRB Approved Form}

https://nugrant.unl.edu/irb/viewPrintedMessage.php?|D $=83988$

10/05/11 2:16 PM

\section{Nebraska

April 28, 2011

Yue Qiu Yu

Department of Educational Psychology

1300 G St Apt 302 E Lincoln, NE 68508

Ian Newman

Department of Educational Psychology

232 TEAC, UNL, 68588-0345

IRB Number: 20110411574EP

Project ID: 11574

Project Title: Chinese Women's Alcohol Expectancy

Dear Yue Qiu:

This letter is to officially notify you of the approval of your project by the Institutional Review Board (IRB) for the Protection of Human Subjects. It is the Boards opinion that you have provided adequate safeguards for the rights and welfare of the participants in this study based on the information provided. Your proposal is in compliance with this institutions Federal Wide Assurance 00002258 and the DHHS Regulations for the Protection of Human Subjects (45 CFR 46). Your project was approved as an Expedited protocol, category 7.

Date of EP Review: 04/15/2011

You are authorized to implement this study as of the Date of Final Approval: 04/28/2011. This approval is Valid Until: 04/27/2012.

We wish to remind you that the principal investigator is responsible for reporting to this Board any of the following events within 48 hours of the event:

* Any serious event (including on-site and off-site adverse events, injuries, side effects, deaths, or other problems) which in the opinion of the local investigator was unanticipated, involved risk to subjects or others, and was possibly related to the research procedures;

* Any serious accidental or unintentional change to the IRB-approved protocol that involves risk or has the potential to recur;

* Any publication in the literature, safety monitoring report, interim result or other finding that indicates an unexpected change to the risk/benefit ratio of the research;

* Any breach in confidentiality or compromise in data privacy related to the subject or others; or

* Any complaint of a subject that indicates an unanticipated risk or that cannot be resolved by the research staff.

For projects which continue beyond one year from the starting date, the IRB will request continuing review and update of the research project. Your study will be due for continuing review as indicated above. The investigator must also advise the Board when this study is finished or discontinued by completing the 
enclosed Protocol Final Report form and returning it to the Institutional Review Board.

If you have any questions, please contact the IRB office at 472-6965.

Sincerely,

Whian G.Thomento

William Thomas, Ph.D.

Chair for the IRB

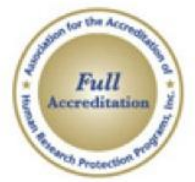




\section{Appendix B}

\section{Pilot Study - Interview Samples}

May 9, 2010 8:00PM-9:30PM Interviewers' brief demographic background: They are from China Shanxi, Xian city (中国陕西省西安市). Dr. Lei is a retired surgeon and Mrs. Liu is a retired hospital worker.

\section{A.你是否經常飲酒? 在哪裡經常掲酒? 什麼時候開始掲酒?}

How often do you consume alcohol?

Where do you consume alcohol often?

When did you start to drink?

With whom do you often drink?

Dr. Lei started to drink when he was in middle school. He picked up the habit by himself. Mrs. Liu never drink and she said that no women drink. Instead of drinking alcohol, women drink coca cola (pop) and Sprite. Dr. Lei said that men drink beer a lot at dinner table, at a feast, a birthday party, a spring festival, a wedding, a baby celebration 滿月酒 (this is a special Chinese celebration for when the baby becomes three months old), a funeral, friend and neighbor gathering, etc. He said that men rarely drink spirit (white wine白酒), but drink beer instead (i.e., Bud Light). Sometimes, young women only drink a little beer for a special event (1/2 cup), Mrs. Liu said.

B.Men and women's questions:

你認為女性在負責酒精消費呢?

How do you think women feel about alcohol consumption?

Dr. Lei and Mrs. Liu: Women in China will not drink

\section{你認為婦女通常在哪裡掲酒?}

Where do you think women would drink alcohol?

In business place, but not at home, in order to build relationships with some government officials

C. Three questions

1. 對女性飲酒的問題,你的主意是什麼? What do you think about women drinking alcohol?

\section{2. 女性在哪裡經常掲酒? Where do women drink alcohol?}

\section{3. 女性什麼時候開始掲酒? When do women start to drink alcohol?}

1, 2, and 3 Answers: Dr. Lei said 陕西 Shanxi women will not drink, no matter how young or old they are. However, he said that only women who work in a business career of public relations (i.e., mediators) will drink at dinner table with males. He also mentioned that women would not drink in 陕西 because of the pressures of feudal traditions dating back to the Qing dynasty. For instance, women could not eat at the same table with men together. In addition to this, the wife did not have the right to eat with her husband. Those 陕西 women who work in the public relations will drink at restaurants and while making a business trade.

Mrs. Lui also said those women drink because they were trained to drink wisely. Women start to drink after they started work in public relations within a business field. For instance, they put a bottle of beer in front of a cup of Sprite. When toasting, they will drink, then they will have a small sip of Sprite after toasting. Actually, they spit the alcohol out to the Sprite cup (We do not know). Moreover, Mrs. Lui and Dr. Lei said that building relationship in China is very important. Women drink because they can mediate and build a good relationship with other companies so that other companies can sign contrasts through women's drinking and socialized eating.

\section{D.你有沒有看到任何酒的廣告? Have you seen any ads for alcohol? 你通常在哪裡看到酒的廣告?}


Dr. Lei and Mrs. Liu: Absolutely, There are two famous alcohol ads which are as follows: 茅台酒 and 五粮液. Male models are dominant in alcohol ads; female models are dominant in make-up ads.

Dr. Lei and Mrs. Liu: magazines and TV everywhere in cities. 


\section{Appendix C}

\section{Samples of Women's Alcohol Expectancies}

If I drink alcohol, I will...

Psychological needs:

Release from anger

relax myself because I have been at work/home entire day

show love passion to others

show my wife or husband best love

overcome from anxiety

make my boss or supervisor or colleagues happy

be in a high person

respect friends or family members surrounding me

be an openness person

be in an update person

be in an in person

feel myself respected

feel myself attractive

feel myself more independent

be a honest person

be in a high cast

be playful

be a fun person among others

be myself as own personality values

be a successful woman

show others I am a woman who knows how to taste Jiu

forget my sadness of a broken relationship with boyfriends or girlfriends

forget a burden relationship with husband

feel good at myself

feel confident

feel men is stupid

release myself from heavy school work

release stress from job hunting

release emotional feelings from relationship between colleagues at work or school

express my feelings to an un-communicated situation from family and friends

have more energy on working and doing school work

be in loved, no more loneliness

express my anger to fight with my boyfriends without any reasons

express my regressive behaviors to others or to society without any logical reasons

think nothing myself

have an emptiness mind

have a quiet moment being myself

hide true self to others or friends or family members in which they do not know my sadness or emotional feelings

feel a moment of fulfillment

be more operational

be better parents

be better lover

live in a fantasy world

be livelier

be spiritual

be enjoyment of the puzzling

show others my maturity

be compassionate at work, studying and social interactions 
be a creative person

be a fashion person

have a moment of simplistic

be a dreamer

show with others or friends or family members my honesty

love

hope

share feelings with others or friends or family members faulty reasoning among women

share feelings with others or friends or family members inflexibility among women

be comforter for others who have a sadness situation

be a responsible parent

be an enthusiastic person who can help others

keep my drinking habit

decrease my boring feelings

taste the bitterness in my life with others

have a moment of dearly drinking

have a passive behaviors

let others know what a strong person I am

let others know what a poor person I am

be a hot person

be a sexy person

be a trouble person

have multiple or mixed feelings

be a fair lady

have spicy feeling

be a better wife

have a sharp feelings

have a beautiful moment of paradise feeling

cry

laugh with others without any thinking reality

hate myself

hate others or friends or family members

have a moment of hypnosis myself

be a gentle woman

be a warm woman

be a kind woman

be a moderate woman

be a competitive woman

be a thoughtful woman

have my own character

have a feeling of consciousness and unconsciousness combination

have a good sleep at night

helps me to cope with my depression

help myself to know how to love

help myself to understand how to communicate with others effectively

have a productive feeling

have a moment of effective tricking men through drinking

compete with lying men who pretended drinking well

let men feel guilty

have a romantic feelings

give up myself for the sake of drinking

new experiences in kind of exploration

have curious blend of logic

have productive lives

have a feeling of vulnerable 
Identify who I am in the world

Become more sexy while having sexual activities with men

show a feeling of solitary and confirm manner in front of men

become more deeply thoughtful

have festive mood

become a unique woman without any reasons

be a very important woman among women and men

import a fashion among women

have an excuse to be crazy

have an excuse to be in love

have a sweet feeling

have a sour feeling

have a bitter feeling

give up myself to enjoy the moment of being in heaven

have a flying feeling

have a swing feeling

have a feeling of being looser

have a feeling of being a victor

punish others to drink for incautious comments

become more talkative

fulfill my sexual and regressive needs

see bright sky in my heart

see a new world

feel no common

feel teary without any commons

have a feeling of miss

fulfill my exploration in which observing others drinking behaviors

have a safe feeling from drinking

overcome my fears

\section{Social needs:}

Meet a man who loves me

Meet new friends

Expand my friend circles

Have exciting experiences with boyfriends

Have sex with men for one-night stands

Meet girlfriends and boyfriends

enjoy friendship gathering

have fun with friends

catch up with friends in bars or nightclubs

have men to buy me drink and play with me

have an exciting meeting with wealthy people in a new places

have a wonderful night with men who I do not know in my life

give myself more chances to meet people to fulfill my social interaction needs

expand my circle of life

have places to entertain with friends

have more time to talk with friends

disucss social topics with others freely in public

have time to explore social night life

have supportive friendships outside of school

build personal relationship with others who I do not know

hang out with friends for a purpose

learn how to play with friends

practice singing with friends or others

learn how to dance with friends 
learn a high cast drinking expectation from friends

encourage friends to be faithful via friendship

love freely with husband

reconnect with society after being married

providing myself a joyful activity among friends

explore social drinking behaviors being an anthologist

have romantic relationship with friends

find someone to talk to

have nothing to do in life, only meeting with people

understand what other drinkers drinking behaviors

meet women from clubs for wealthy women

show a mercy with others in social setting

learn how to social with others

look for friends' advices on how to solve my own problems

look for friends who can care about my feelings

share my stories freely with others in public

share my opinions about dealing with working situation, family situation or colleagues situation

listen to friends' stories nearly

listen to others' life experiences

understand how society is function daily in a community through circles of friends' friends

learn social norms from others

meet people to overcome my unsafe feelings

have night life productively with people

have an wonderful night with people after a hard working day

investigate what men need in their lives through social drinking

Learn from what in topics modern women usually talk about in the community

expand worldviews about characters of my friends or others

learn from what updates are there out in the society, such as songs, music, singers, dancers, fashion or world news

have a sense of understanding of other drinkers' feelings

find people who are as same as me in common

exchange my views freely on values or virtues or morals with others

share with others about my life at school, home or work

exchange worldviews or cultural perspectives within groups

look for intimate relationship

have more friends supporting me to my situation

\section{Family needs:}

celebrate I got hired by a company or school or a organization

get acquainted with old friends who I have not seen for a long time through union event

celebrate birthdays with family members and friends

show my regrets at funerals to the one who passed away, such as friend, love ones, family members...

celebrate getting married at wedding

celebrate at anniversary

respect a ritual or religious culture

celebrate at graduation party

celebrate at farewell party

be happy with families gathering

show respect to family traditional

share my love with family or friends at any kind of unions

celebrate Chinese New Year, Mid-Autumn day, National day, and etc.

\section{Economic needs:}

make money for myself

make money and raise my family 
make money and support parents

sell alcohol accordingly make extra money in my life

make extra money so that I will become wealthy

become professional drinkers to make extra money

become professional drinkers due to a poor family background

drink with customers

play with customers

sing with customers

attract customers to come to consume in bars or nightclubs in order to increase the business for myself

become a successful woman in business field, such as professionals in public relationships

overcome my barriers in business field

satisfy my supervisors at a business drinking dinner table

respect other colleagues who toast me at dinner table

not avoid some kinds of political forces

hide secret of kinds of situations

tell others that I am in high class

understand how to conduct business

make a living in society under many occasions

become a professional drinker to entertain others in public services, in plus, I can negotiate with customers to buy westerns drinks

be more responsible to my job to earn extra money, such as "selling good drug"

have extra money to buy good quality make-ups, in fashions, organic food, and to eat in high class

restaurants or to enjoy life

learn how to self-control while drinking at business dinner table with officials

sell alcohol and make extra money to spend "social" time with customers

show my capabilities in business filed

skip schools to earn fast money and look for fun

\section{Health needs:}

Become more beautiful

Have long lives

Be sexier

Look good as young as I am

be in

be high

expect a healing of diseases, such as arthritis

get benefit from alcohol nutrition

help increase blood flow

prevent cancer

feel well

be more energized at work and school

have a healthy life

reduce heart disease

feel Irritated

Feel Uncomfortable

Get Dizzy

Loose temper

Feel hopeless in the future

\section{Other needs:}

drink with partner with different attitudes, such as the attitudes are drinking nothing or drink too much shout out for a black/brother sharp type against normal

increase my self-efficacy to believe that I have a capability at school and work or in society

be a strong ferniest

look for gender equality 
be a brilliant woman in society

trick men

call for playful

call for a protest

be in a high cast position

increase my expectation of drinking from other drinking cultures' influences

Against the mess society

call for democracy for women 


\section{Appendix D}

\section{6 - Items Alcohol Expectancy Statements}

\section{Psychological Needs:}

\section{Theme 1: relief of anxiety and emotional behaviors}

1. If I drink, then it will help me release my anger.

2. If I drink, then it will help me overcome anxiety.

3. If I drink, I will be able to forget the sadness of a broken relationship.

4. If I drink, then it will help me release emotions from relationships with others (colleagues or workmates).

5. If I drink, it will relieve my stress.

6. If I drink, it will relieve my boredom.

7. If I drink, it will help me define or identify who I am.

8. If I drink, then I will be more relaxed.

\section{Theme 2: Defining or Redefining one's image or values}

9. If I drink, then I will feel respected.

10. If I drink, then I will feel attractive.

11. If I drink, then I will feel like and independent person.

12. If I drink, I will feel good about myself.

13. If I drink, then I will feel superior to others. (other men or women)?

14. If I drink, then I will feel more mature.

15. If I drink, then my self-efficacy will increase.

16. deleted

17. If I drink, then I will have the confidence to follow fashion trends.

18. If I drink, then I will be a more successful businessperson and make my supervisor and colleagues happy.

19. If I drink, then I will feel I am a gentle and elegant person (woman or man).

20. If I drink, then I will be a more open and talkative person.

21. If I drink, then I will be a more playful and fun person.

22. If I drink, then others will see I am a person who knows how to taste Jiu.

23. If I drink, then I will be a more responsible partner because I will understand my partner's drinking experiences.

24. If I drink, it helps me to be a better lover.

25. If I drink, then I will be livelier in social drinking settings.

26. If I drink, then I will be more honest.

27. If I drink, I will be able to enjoy life's mysteries without having to solve them.

28. If I drink, then I will be more creative. 
29. If I drink, I will feel I am a dreamer (or if I drink, then I will feel like a person who dose nothing but dream).

30. If I drink, I will be able to help comfort other drinkers who are sad (or If I drink, then I will be warmer towards other).

31. If I drink, then I will be more enthusiastic about helping others.

32. If I drink, then I will feel sexy.

33. If I drink, then I will have a "bad girl" or "bad boy" image.

34. deleted

35. deleted

36. deleted

37. If I drink, then I will be more competitive.

38. If I drink, then I will be more thoughtful.

39. If I drink, then I will not have to justify myself to others.

\section{Theme 3: self- expression}

40. If I drink, then I will be more passionate with my spouse or another person.

41. If I drink, then I will be more confident in front of others.

42. If I drink, then I will be able to have fun tricking others into drinking more than me.

43. If I drink, it will become my drinking habit.

44. If I drink, then I will feel able to compete with other drinkers.

45. If I drink, then I will be able to ignore reality and just be happy with others.

46. If I drink, then I will be able to embarrass others who lie about being able to drink well.

\section{Theme 4: Sharing others' perspectives (perspective taking)}

47. If I drink, then I will be able to confidently talk with someone whom I have nothing else in common with.

48. If I drink, then I will be allowed to cry without being judged.

49. If I drink, then I will be able to discuss my life with friends.

50. If I drink, then it will help me see other perspectives and better communicate with others.

51. If I drink, it will help me to know myself better how to love, no more loneliness.

52. If I drink, then it will let others know that I am a strong person.

53. If I drink, then I will be able to let others know that I am a poor (weak) person.

\section{Theme 5: positive- feelings}

54. If I drink, then I will have a quiet moment in which to be myself.

55. If I drink, I will have a moment of drinking enjoyment.

56. If I drink, I will have an excuse to be in love.

57. If I drink, then I will get a good night's sleep. 
58. If I drink, then I will be able to imagine living in a fantasy world.

59. If I drink, then I will be able to share with others, friends, or family about my hidden feelings about women's traditional roles.

60. If I drink, I will be able to stand up for those who go against tradition.

\section{Social Needs/Sexual needs:}

\section{Theme 1: meet lovers}

61. If I drink, then it will help me fulfill my sexual needs.

62. If I drink, it will help me to fulfill my exploration of drinking in which observing other drinking behaviors

63. If I drink, then my sexual performance will improve.

64. If I drink, then I will be able to have great one-night stands.

\section{Theme 2: expand circle of friends (girlfriends or boyfriends)}

65. If I drink, then I will be able to understand or accept others more easily.

66. If I drink, then I will explore more drinking entertainment options with friends or strangers.

67. If I drink, then I will develop supportive friendships outside of school.

68. If I drink, then I will have romantic relationships with my friends.

69. If I drink, then I will feel like I can understand other drinkers' feelings.

\section{Theme 3: explore entertainments with friends or strangers}

70. If I drink, then friends will buy me drinks and entertain me.

71. If I drink, then I will be able to meet wealthy people.

72. If I drink, then I will be able to explore the social nightlife.

73. If I drink, it will help me communicate with others about coping with work and family problems.

74. If I drink, then I will be able to have fun with people at night after a day of hard work.

\section{Theme 4: learning experiences among friends of friends}

75. If I drink, I will be able to explore different kinds of drinking experiences

76. If I drink, then I will understand how society functions.

77. If I drink, then I will be more in tune with popular culture or be caught up on pop culture and world news.

78. If I drink, it will help me pursue gender equality.

79. If I drink, then I will be able to share my stories freely with others in public.

80. If I drink, then I will be able to look for friends who care about my feelings.

81. If I drink, it will help me exchange worldviews or cultural perspectives within groups of different.

82. If I drink, it will help me reconnect with society after being married.

\section{Family needs:}


83. If I drink, I will have more passion to celebrate holidays and special events.

\section{Theme 1: maintain friends/family traditions}

84. If I drink, it will help me respect and socialize better with family and friends at reunions.

85. If I drink, I will be able to get reacquainted with old friends.

\section{Economic needs:}

\section{Theme 1: make money for living due to poverty}

86. deleted

\section{Theme 2: make money becoming professional drinkers}

87. If I drink, then it will help me overcome barriers in the business world.

88. If I drink, then I will show respect to others who toast me at the dinner table.

89. If I drink, then I will have self-control while drinking during business dinners.

90. deleted

\section{Health needs}

\section{Theme 1: good looking due to social influences}

91. If I drink, it will help me appear better-looking and popular.

\section{Theme 2: remedy}

92. If I drink, it will help to cure my arthritis.

93. If I drink, it will help to prevent cancer.

94. If I drink, I will get a nutritional benefit from the alcohol.

95. If I drink, then I will be healthier (i.e. increase blood flow).

96. If I drink, I will be at less risk for heart disease.

\section{Theme 3: Negative Thoughts}

97. If I drink, then I will feel dizzy

98. If I drink, then I will feel more irritated

99. If I drink, then I will lose my temper.

100. If I drink, then I will feel hopeless about my future. 
Appendix E

\title{
Women's Alcohol Expectancy Instrument (82-items) in English and Chinese version
}

\author{
College Students' Alcohol Expectancy Survey \\ [English translation from Mandarin]
}

This survey is about alcohol use and the effects of drinking alcohol on a person. The information you provide will be used to develop better health education for young people. Your help is greatly appreciated!

Before you begin, please read the following instructions carefully:

1) This is not an exam or a test. Please answer each question according to your actual behaviors and thoughts. There are no right or wrong answers. How and whether you answer all the questions will not affect your grade in this class or your standing in the university.

2) Do not write your name on this survey. The answers you give will be kept private. No one including your teachers will know what you have written. The questions that ask about your background will be used only to describe the types of students completing this survey. The information will not be used to identify you.

3) There are no hidden meanings in the questions or responses. Please answer the questions according to your first thought.

4) You may omit any questions that you choose not to answer.

5) If you are not clear about the meaning of any question, leave the answer blank.

6) The survey will take less than 20 minutes to complete and includes ninety-two items.

Thank you so much for your participation!

Part 1. Indicate how strongly you agree or disagree with each statement that describes what happens when a person drinks alcohol by circling a number on a 5-point scale like this:

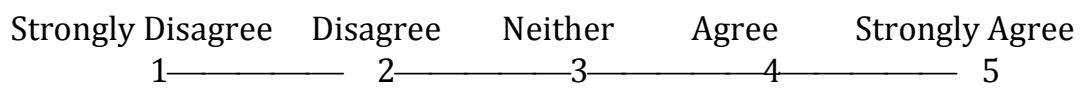

Please circle one number for each statement that best reflects your belief.

1. If I drink, it will help me to release from my anger

2. If I drink, it will help me to overcome anxiety

3. If I drink, I will forget my sadness of a broken relationship

4. If I drink, it will help me to release my emotions from relationship with work mates

5. If I drink, it will help me to release my stress

6. If I drink, I will decrease my boredom

7. If I drink, it will help me define or identify who I am.

8. If I drink, then I will be more relaxed

9. If I drink, then I will feel respected by other people

10. If I drink, then I will feel attractive 
11. If I drink, then I will feel I like a more independent person

12. If I drink, then I will feel good about myself

13. If I drink, then I will feel superior to other people

14. If I drink, then I will feel more mature

15. If I drink, then my self-efficacy will increase

16. If I drink, then I will be a more successful business person

17. If I drink, then I will feel I am a more gentle and elegant person

18. If I drink, then I will be a more open and talkative person

19. If I drink, then I will be a more playful and fun person

20. If I drink, then I will be a more responsible partner.

21. If I drink, it helps me to be a better lover

22. If I drink, then I will be livelier at social drinking occasions

23. If I drink, then I will be more honest

24. If I drink, then I will be able to enjoy life's mysteries without having to solve them

25. If I drink, then I will be more creative

26. If I drink, I will feel I am a dreamer

27. If I drink, then I will be warmer toward other people

28. If I drink, then I will be more enthusiastic about helping other people

29. If I drink, I will feel sexy

30. If I drink, then I will have be a "bad boy" or "bad girl" image

31. If I drink, then I will feel more competitive

32. If I drink, then I will feel more thoughtful

33. If I drink, then I will feel no need to explain or justify myself to other people

34. If I drink, then I will be more confident in front of other people

35. If I drink, it will become my drinking habit

36. If I drink, then I will be able to ignore reality and just be happy with other people

37. If I drink, then I will be able to talk confidently with people whom I have nothing in common with

38. If I drink, then I will be allowed to cry without being judged

39. If I drink, then I will be able to discuss my life with friends

40. If I drink, then it will help me to see other people's perspectives and communicate better with other people

41. If I drink, then it will let other people know that I am a strong person

42. If I drink, then I will have a quiet moment in which to be myself

43. If I drink, then I will a moment of drinking enjoyment

44. If I drink, then I will have an excuse to be in love

45. If I drink, then I will get a good night's sleep

46. If I drink, then I will be able to imagine living in a fantasy world

47. If I drink, then I will be able to share my thoughts with other people, friends, or family

48. If I drink, then I will be able to stand up for other people who go against tradition

49. If I drink, then it will help me to fulfill my sexual needs

50. If I drink, then it will help me to fulfill my exploration of drinking by observing other people's drinking behaviors

51. If I drink, then my sexual performance will improve

52. If I drink, then I will be able to understand and accept other people more easily

53. If I drink, then I will have more entertainment options

54. If I drink, then I will develop supportive friendships outside of school

55. If I drink, then I will feel like I can understand other people's feelings

56. If I drink, then I will be able to meet wealthy people

57. If I drink, then I will be able to explore the social nightlife.

58. If I drink, it will help me communicate with other people about coping with work and family problems

59. If I drink, then I will be able to have fun with other people at night after a hard work day

60. If I drink, then I will be able to explore different kinds of drinking experiences

61. If I drink, then I will understand how society functions 
62. If I drink, then I will be more in tune with popular culture

63. If I drink, then it will help me to pursue gender equality

64. If I drink, then I will be able to share my stories freely with others in public

65. If I drink, then I will be able to look for friends who can care about my feelings

66. If I drink, then it will help me to exchange world views or cultural perspectives with people from different groups

67. If I drink, it will help me reconnect with society after being married

68. If I drink, then I will have more passion to celebrate holidays and special events

69. If I drink, it will help me social better with family and friends at reunions

70. If I drink, I will be able to get re-acquainted with old friends who I have not seen for a long time

71. If I drink, then I will be able to overcome the barriers in business field

72. If I drink, then I will be showing respect to other people who toast me at dinner table

73. If I drink, then I will learn self-control while drinking at business dinner table

74. If I drink, then it will help me to appear better looking and popular

75. If I drink, it will help me to prevent cancer

76. If I drink, I will get a nutritional benefit

77. If I drink, it will increase my blood flow

78. If I drink, I will be at less risk for heart disease

79. If I drink, then I will feel dizzy

80. If I drink, then I will feel more irritated

81. If I drink, then I will lose my temper

82. If I drink, then I will feel hopeless about my future.

Part 2: This part of the questionnaire asks about alcohol use. In this questionnaire "alcohol" refers to beer, liquor, wine, fruit wine, rice wine, horse milk wine, or any other beverage that contains alcohol. For this part, choose the one answer to each question that best describes you or your class.

83. On how many days did you drink alcohol in the past 12 months from today?

(1) I never drank alcohol

(2) I did not drink any alcohol in the past 12 months

(3) I drank alcohol in the past 12 months, did not drink in the past 30 days

(4) I drank alcohol in the past 30 days

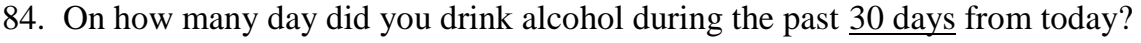

(1) I never drank alcohol

(2) I did not drink alcohol in the past 30 days

(3) I drank alcohol on 1 to 3 days of the past 30 days

(4) I drank alcohol on 4 to 9 days of the past 30 days

(5) I drank alcohol on 10 to 20 days of the past 30 days

(6) I drank alcohol on at least 20 days up to almost every day of the past 30 days

85. Think about the male students at your university who are in the same class as you. On how many days during a typical month do you think they drink?

(1) The male students in my university class never drink alcohol

(2) The male students in my university class do not drink in a typical month

(3) The male students in my university class drink alcohol on 1 to 3 days of a typical month

(4) The male students in my university class drink alcohol on 4 to 9 days of a typical month

(5) The male students in my university class drink alcohol on 10 to 20 days of a typical month

(6) The male students in my university class drink alcohol on at least 20 days or more in a typical month

86. Think about the female students at your university who are in the same class as you. On how many days during a typical month do you think they drink? 
(1) The female students in my university class never drink alcohol

(2) The female students in my university class do not drink in a typical month

(3) The female students in my university class drink alcohol on 1 to 3 days of a typical month

(4) The female students in my university class drink alcohol on 4 to 9 days of a typical month

(5) The female students in my university class drink alcohol on 10 to 20 days of a typical month

(6) The female students in my university class drink alcohol on at least 20 days or more in a typical month

87. Think about the male students at your university who are in the same class as you. On how many days during a typical year do you think they drink?

(1) The male students in my university class never drink alcohol

(2) The male students in my university class do not drink alcohol in a typical year

(3) The male students in my university class drank alcohol in the past 12 months, but did not drink in the past 30 days

(4) The male students in my university class drank alcohol in the past 30 days

88. Think about the female students at your university who are in the same class as you. On how many days during a typical year do you think they drink?

(1) The female students in my university class never drink alcohol

(2) The female students in my university class do not drink alcohol in a typical year

(3) The female students in my university class drank alcohol in the past 12 months, but did not drink in the past 30 days

(4) The female students in my university class drank alcohol in the past 30 days

Part 3. These questions are about you. Please choose the one answer for each question that best describes you.

89. Age

90. Gender: Male Female

91. Grade: Freshmen Sophomore Junior Senior Graduate

92. Where is your hometown?

(1) State-ruled city

(2) Province capital

(3) District city

(4) County city

(5) Town or rural area

大学生问卷调查

(二)

这项调查是关于饮酒行为和酒精对人的影响。您提供的信息将会对大学生健康教育提供帮助。

在开始回答问题之前, 请您仔细阅读以下说明：

1）这不是考试或测验,

没有所谓的正确答案. 您是否回答, 以及具体的答案不会影响您的成绩和在学校的表现. 
2）请不要在问卷上填写您的名字. 您的回答将会完全保密. 您身边没有人会知道您的答案, 包括 老师在内. 有关您个人背景的问题只是用于描述参与本调查的人群特征, 不会用来识别您的 身份.

3）所有的问题都没有隐藏的含义. 请您根据第一反应来作答, 不必花费时间去考虑您的选择.

4) 填写问卷时, 请不要跟同学交谈, 也不要参考别人的答案. 如果您不明白某个问题, 请留下空 白.

5）请检查确认没有问题遗漏. 当您完成后, 请跟随问卷调查者的指示.

谢谢您的合作！

\section{第一部分: 对下面的每个问题, 请表明你的看法, 你的看法可分为五个等级:}

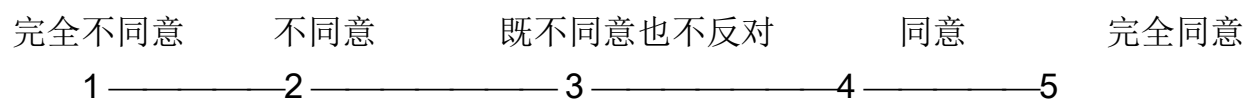

\section{请在代表你看法的数字上打圈 “O”。}

\begin{tabular}{|c|c|c|c|c|c|}
\hline & $\begin{array}{c}\text { 完全 } \\
\text { 不同 } \\
\text { 意 }\end{array}$ & $\begin{array}{c}\text { 不同 } \\
\text { 意 }\end{array}$ & $\begin{array}{l}\text { 既不 } \\
\text { 同意 } \\
\text { 也不 } \\
\text { 反对 }\end{array}$ & 同意 & $\begin{array}{l}\text { 完全 } \\
\text { 同意 }\end{array}$ \\
\hline 1. 如果我喝酒, 酒能释放我的愤怒。 & 1 & 2 & 3 & 4 & 5 \\
\hline 2. 如果我喝酒, 我会暂时克服生活上的焦虑。 & 1 & 2 & 3 & 4 & 5 \\
\hline 3. 如果我喝酒, 我会忘记与男（女）朋友或配偶分手的悲伤。 & 1 & 2 & 3 & 4 & 5 \\
\hline 4. 如果我喝酒, 我会舒缓与同学或同事之间关系带来的压力。 & 1 & 2 & 3 & 4 & 5 \\
\hline 5. 如果我喝酒, 我会释放压力。 & 1 & 2 & 3 & 4 & 5 \\
\hline 6. 如果我喝酒, 我会打发无聊。 & 1 & 2 & 3 & 4 & 5 \\
\hline 7. 如果我喝酒, 我会重新思考（认识）自我价值。 & 1 & 2 & 3 & 4 & 5 \\
\hline 8. 如果我喝酒, 我会放松自己。 & 1 & 2 & 3 & 4 & 5 \\
\hline 9. 如果我喝酒, 我会感到自己被尊重。 & 1 & 2 & 3 & 4 & 5 \\
\hline 10. 如果我喝酒, 我会感到自己有吸引力。 & 1 & 2 & 3 & 4 & 5 \\
\hline 11. 如果我喝酒, 我会感觉自己更独立。 & 1 & 2 & 3 & 4 & 5 \\
\hline 12. 如果我喝酒, 我会自我感觉轻松愉快。 & 1 & 2 & 3 & 4 & 5 \\
\hline
\end{tabular}




\begin{tabular}{|c|c|c|c|c|c|}
\hline $\begin{array}{l}13 . \\
\text { 如果我喝酒, 我会肯定自己的地位, 甚至得到超越其他人的优越 } \\
\text { 感。 }\end{array}$ & 1 & 2 & 3 & 4 & 5 \\
\hline 14. 如果我喝酒，我会拥有片刻成就感。 & 1 & 2 & 3 & 4 & 5 \\
\hline $\begin{array}{l}15 . \\
\text { 如果我喝酒, 我能树立信心并相信自己能胜任学校或工作中的任 } \\
\text { 务。 }\end{array}$ & 1 & 2 & 3 & 4 & 5 \\
\hline $\begin{array}{l}16 . \\
\text { 如果我喝酒, 我会感到自己是个成功的人士, 深受老板同事喜欢 } \\
\text { 。 }\end{array}$ & 1 & 2 & 3 & 4 & 5 \\
\hline 17. 如果我喝酒, 我会感到自己优雅有气质。 & 1 & 2 & 3 & 4 & 5 \\
\hline 18. 如果我喝酒, 我会感到自己是个开放, 健谈的人。 & 1 & 2 & 3 & 4 & 5 \\
\hline 19．如果我喝酒，我会感到自己是个有趣的人。 & 1 & 2 & 3 & 4 & 5 \\
\hline $\begin{array}{l}20 . \quad \text { 如果我喝酒, 我会感到自己是个更有责任心的配偶, } \\
\text { 因为我能理解我的配偶饮酒经历。 }\end{array}$ & 1 & 2 & 3 & 4 & 5 \\
\hline \multirow[t]{2}{*}{ 21. 如果我喝酒，我会感到自己是个更好的情人。 } & 1 & 2 & 3 & 4 & 5 \\
\hline & $\begin{array}{l}\text { 完全 } \\
\text { 不同 } \\
\text { 意 }\end{array}$ & $\begin{array}{c}\text { 不同 } \\
\text { 意 }\end{array}$ & $\begin{array}{l}\text { 既不 } \\
\text { 同意 } \\
\text { 也不 } \\
\text { 反对 }\end{array}$ & 同意 & $\begin{array}{l}\text { 完全 } \\
\text { 同意 }\end{array}$ \\
\hline 22. 如果我喝酒, 我会感到自己是个有活力的人。 & 1 & 2 & 3 & 4 & 5 \\
\hline 23. 如果我喝酒, 我会感到自己是个诚实的人。 & 1 & 2 & 3 & 4 & 5 \\
\hline 24. 如果我喝酒, 我会不再计较生活中无法理解的事情和难题。 & 1 & 2 & 3 & 4 & 5 \\
\hline 25. 如果我喝酒, 酒能激发我更多的灵感。 & 1 & 2 & 3 & 4 & 5 \\
\hline 26. 如果我喝酒，我会感到自己是一个梦想家。 & 1 & 2 & 3 & 4 & 5 \\
\hline $\begin{array}{l}27 . \\
\text {. 如果我喝酒, 我会与朋友一起喝, 而且会安慰那些悲伤的喝酒人 }\end{array}$ & 1 & 2 & 3 & 4 & 5 \\
\hline $\begin{array}{l}28 . \\
\text { 如果我喝酒, 我会感到自己是一个很仗义的愿意帮助别人的人。 }\end{array}$ & 1 & 2 & 3 & 4 & 5 \\
\hline 29. 如果我喝酒, 我会感到自己是个性感的人。 & 1 & 2 & 3 & 4 & 5 \\
\hline 30. 如果我喝酒, 我会担心别人认为我行为不检。 & 1 & 2 & 3 & 4 & 5 \\
\hline 31. 如果我喝酒, 喝酒激发我感到自己是个有竞争力的人。 & 1 & 2 & 3 & 4 & 5 \\
\hline 32. 如果我喝酒, 喝酒激发我更好地思考。 & 1 & 2 & 3 & 4 & 5 \\
\hline 33. 如果我喝酒, 我能隐藏我的内心世界。 & 1 & 2 & 3 & 4 & 5 \\
\hline 34. 如果我喝酒, 我会在别人面前显示我的自信。 & 1 & 2 & 3 & 4 & 5 \\
\hline 35. 如果我喝酒，喝酒会成为我的习惯。 & 1 & 2 & 3 & 4 & 5 \\
\hline 36. 如果我喝酒, 我能得到片刻的开心, 从而逃离现实。 & 1 & 2 & 3 & 4 & 5 \\
\hline
\end{tabular}




\begin{tabular}{|c|c|c|c|c|c|}
\hline 37. 如果我喝酒, 我可以和没有共同点的人交谈。 & 1 & 2 & 3 & 4 & 5 \\
\hline 38. 如果我喝酒, 我能允許自己放声大哭。 & 1 & 2 & 3 & 4 & 5 \\
\hline 39. 如果我喝酒, 我会与朋友一起谈论生活。 & 1 & 2 & 3 & 4 & 5 \\
\hline 40. 如果我喝酒, 我会听取别人的看法, 有效与他人沟通。 & 1 & 2 & 3 & 4 & 5 \\
\hline 41. 如果我喝酒, 我会展示我坚强的一面。 & 1 & 2 & 3 & 4 & 5 \\
\hline 42. 如果我喝酒, 我会感到自己拥有片刻的平静做回自己。 & 1 & 2 & 3 & 4 & 5 \\
\hline 43. 如果我喝酒, 我会享受饮酒的新感觉片刻时光。 & 1 & 2 & 3 & 4 & 5 \\
\hline 44. 如果我喝酒, 我会有一个借口去恋爱, 去疯狂。 & 1 & 2 & 3 & 4 & 5 \\
\hline 45．如果我喝酒，我会拥有一个良好睡眠。 & 1 & 2 & 3 & 4 & 5 \\
\hline 46. 如果我喝酒, 我会活在一个梦幻世界。 & 1 & 2 & 3 & 4 & 5 \\
\hline \multirow{2}{*}{$\begin{array}{l}\text { 47. 如果我喝酒, 我会与朋友, 家人, 或他人交流对女（男）性 } \\
\text { 的错误守旧的看法. }\end{array}$} & 1 & 2 & 3 & 4 & 5 \\
\hline & $\begin{array}{l}\text { 完全 } \\
\text { 不同 } \\
\text { 意 }\end{array}$ & $\begin{array}{c}\text { 不同 } \\
\text { 意 }\end{array}$ & $\begin{array}{l}\text { 既不 } \\
\text { 同意 } \\
\text { 也不 } \\
\text { 反对 }\end{array}$ & 同意 & $\begin{array}{l}\text { 完全 } \\
\text { 同意 }\end{array}$ \\
\hline $\begin{array}{l}48 . \\
\text { 如果我喝酒, 我会感到自己是个打破传统思想与众不同的人。 }\end{array}$ & 1 & 2 & 3 & 4 & 5 \\
\hline 49. 如果我喝酒，我会感到自己找回往日的激情。 & 1 & 2 & 3 & 4 & 5 \\
\hline 50. 如果我喝酒, 我对喝酒的好奇心会得到满足。 & 1 & 2 & 3 & 4 & 5 \\
\hline 51. 如果我喝酒, 我会与男 (女) 朋友有更多的激情。 & 1 & 2 & 3 & 4 & 5 \\
\hline 52. 如果我喝酒, 我会从新的角度认识自己的朋友和他人。 & 1 & 2 & 3 & 4 & 5 \\
\hline 53. 如果我喝酒, 我会更好地与朋友或陌生人一起找乐子。 & 1 & 2 & 3 & 4 & 5 \\
\hline 54. 如果我喝酒, 我会找到校园外的友谊。 & 1 & 2 & 3 & 4 & 5 \\
\hline 55. 如果我喝酒, 我会了解其他饮酒者的感觉。 & 1 & 2 & 3 & 4 & 5 \\
\hline 56. 如果我喝酒, 我会与有钱人玩的很刺激。 & 1 & 2 & 3 & 4 & 5 \\
\hline 57. 如果我喝酒, 我会与朋友一起追求夜生活。 & 1 & 2 & 3 & 4 & 5 \\
\hline $\begin{array}{l}58 . \\
\text { 如果我喝酒, 我会与人交流如何处理工作，家庭，同事之间的问 } \\
\text { 题。 }\end{array}$ & 1 & 2 & 3 & 4 & 5 \\
\hline $\begin{array}{l}\text { 59. 如果我喝酒， 我会辛勤工作一天后， } \\
\text { 享受一个愉快的夜晚。 }\end{array}$ & 1 & 2 & 3 & 4 & 5 \\
\hline 60. 如果我喝酒, 我会有各种不同的经历。 & 1 & 2 & 3 & 4 & 5 \\
\hline 61. 如果我喝酒, 我能通过朋友圈了解社会信息。 & 1 & 2 & 3 & 4 & 5 \\
\hline $\begin{array}{l}62 . \\
\text { 如果我喝酒, 我会了解到现代流行的话题（例如: 音乐, 时尚) }\end{array}$ & 1 & 2 & 3 & 4 & 5 \\
\hline
\end{tabular}




\begin{tabular}{|c|c|c|c|c|c|}
\hline 。 & & & & & \\
\hline 63. 如果我喝酒, 我会寻求男女平等。 & 1 & 2 & 3 & 4 & 5 \\
\hline 64. 如果我喝酒, 我会在公共场所畅所欲谈。 & 1 & 2 & 3 & 4 & 5 \\
\hline 65. 如果我喝酒, 我会有机会寻找可以关心自己的朋友。 & 1 & 2 & 3 & 4 & 5 \\
\hline 66. 如果我喝酒, 我会在群体中与人交流人生价值观, 道德观。 & 1 & 2 & 3 & 4 & 5 \\
\hline 67．如果我喝酒，我会避免在婚后与社会脱节。 & 1 & 2 & 3 & 4 & 5 \\
\hline $\begin{array}{l}68 . \\
\text { 如果我喝酒, 我会让庆祝活动的气氛更好（例如：生日, 婚礼, } \\
\text { 周年纪念, 毕业, 欢送会, 中秋)。 }\end{array}$ & 1 & 2 & 3 & 4 & 5 \\
\hline 69. 如果我喝酒，我能尊重家庭传统并与家人同乐。 & 1 & 2 & 3 & 4 & 5 \\
\hline 70. 如果我喝酒, 我能更好与久未见面的老友叙旧。 & 1 & 2 & 3 & 4 & 5 \\
\hline 71. 如果我喝酒, 我会更好地谈生意。 & 1 & 2 & 3 & 4 & 5 \\
\hline 72. 如果我喝酒, 我能对向我敬酒的人表示尊重。 & 1 & 2 & 3 & 4 & 5 \\
\hline \multirow[t]{2}{*}{$\begin{array}{l}73 . \\
\text { 如果我喝酒, 我会学会在应酬的时候如何控制自己的饮酒量。 }\end{array}$} & 1 & 2 & 3 & 4 & 5 \\
\hline & $\begin{array}{l}\text { 完全 } \\
\text { 不同 } \\
\text { 意 }\end{array}$ & $\begin{array}{c}\text { 不同 } \\
\text { 意 }\end{array}$ & $\begin{array}{l}\text { 既不 } \\
\text { 同意 } \\
\text { 也不 } \\
\text { 反对 }\end{array}$ & 同意 & $\begin{array}{l}\text { 完全 } \\
\text { 同意 }\end{array}$ \\
\hline 74. 如果我喝酒, 我会变得更年轻, 健康, 性感, 时尚。 & 1 & 2 & 3 & 4 & 5 \\
\hline 75. 如果我喝酒, 酒能预防癌症。 & 1 & 2 & 3 & 4 & 5 \\
\hline 76. 如果我喝酒, 我会从酒中获取营养。 & 1 & 2 & 3 & 4 & 5 \\
\hline 77. 如果我喝酒, 酒能加快血液循环。 & 1 & 2 & 3 & 4 & 5 \\
\hline 78. 如果我喝酒, 酒能预防心脏疾病。 & 1 & 2 & 3 & 4 & 5 \\
\hline 79. 如果我喝酒, 我会感觉晕眩。 & 1 & 2 & 3 & 4 & 5 \\
\hline 80．如果我喝酒，我感到更烦躁。 & 1 & 2 & 3 & 4 & 5 \\
\hline 81. 如果我喝酒, 我控制不了自己的脾气。 & 1 & 2 & 3 & 4 & 5 \\
\hline 82. 如果我喝酒, 我会感到自己荒废了美好的前程。 & 1 & 2 & 3 & 4 & 5 \\
\hline
\end{tabular}




\section{第二部分:这部分问卷是有关饮酒的问题.}

这里的 “酒” 包括啤酒, 白酒, 葡萄酒, 果酒, 米酒, 奶酒, 以及任何含酒精的饮料. 每个问题请选择一 个最佳答案.

（6）在过去的一年内, 你大概有多少天喝过酒?

1）从不喝酒

2）过去一年内没喝过酒

3）过去一年内喝过酒，但近一个月没喝过

4）过去一个月喝过酒

（7）在过去的 30 天内, 你大概有多少天喝过酒?

1）从不喝酒

2）在过去 30 天内, 我没喝过酒

3) $1-3$ 天

4) 4-9天

5） $10-20$ 天

6）至少 20 天到几乎每天

85. 想一下你班上的男同学, 你认为一个月他们有多少天喝过酒?
1）从不喝酒
2）不会每个月都喝
3) $1-3$ 天
4) 4-9天
5） 10-20天
6） 20 天或更多

86. 想一下你班上的女同学, 你认为二个月他们有多少天喝过酒?
1）从不喝酒
2）不会每个月都喝
3） $1-3$ 天
4) 4-9天
5) 10-20天
6） 20 天或更多

87. 想一下你班上的男同学, 你认为一年他们有多少天喝过酒?
1）从不喝酒
2）过去一年内没喝过酒
3）过去一年内喝过酒，但近一个月没喝过
4）过去一个月喝过酒

88. 想一下你班上的女同学, 你认为一年他们有多少天喝过酒?
1）从不喝酒
2）过去一年内没喝过酒
3）过去一年内喝过酒，但近一个月没喝过
4）过去一个月喝过酒

（调查结束，再次感谢！) 


\section{第三部分：以下问题请选择一个最佳答案。}

89、年龄

90、性别：男 女

91. 你大学几年级?
1）大一
2) 大二
3）大三
4）大四
5 ) 研究生

92. 你的家乡是?
(1) 直辖市
（2）省会城市
(3) 地级市
(4) 县级
(5) 乡镇

（调查结束, 再次感谢！) 


\section{Appendix F}

\section{Transcribed Interview Samples}

July 3 Dinner in a restaurant at 7:30PM -9:00PM: Three men and three women

Interviewer: What do you think of women who drink alcohol?

Woman A (40 years old): no women drinking ads, but use women to sell alcohol products a lot.

Man 1 (26 years old): normally on TV women drink alcohol

Lady 1 (26 years old): like going out with friends at bars or MTV

Man 2 (26 years old): likes hanging out with friends in bars or KTV outside: It means that you have to

drink beer, otherwise, you will not be happy after drinking.

Lady 2: No...no, only use women to sale those ads because women wear less, and tract more people to buy beers, plus those women are very sexy.

...laughing...

Interviewer: Under which occasion, will you see people increase their alcohol consumption?

Man 2: world cup.... a lot

Man 3: Of course, society is developed, more people have money and more people need nightlife, thus, there are many alcohol products ...you know people have lots of needs, consequently there are lots of night activities to fulfill human beings... what products? such as bars in Kaiping, one opens this week, next week, another bar opens... keep on and on...

*All transcribing notes are based on American Central Time

100621_002 June 2120102127 a Pearl River radio station about elderly people drink can keep healthy

This is a mass media show from a Pearl River radio station. A Chinese doctor shared with audiences on the radio stations about medicinal alcohol.

Doctor: Tong Hao Huang Jing Jiu gives nutrition to kidney, stomach and liver...noise....alternatively, 酒 jiu provides you a healthy benefit. The doctor said that keeping healthy and longevity is not for wealthy people but also for general populations.

100622_002 June 2220101003 in a nightclub

Interviewer: What do women expect through drinking alcohol?

A lady: for making a living .... I am from He Bei and enjoys living in Kaiping.

100622_004 June 2220101106 in a KTV three men and three women drink together at a drinking game table

Interviewer: What do you think of women who drink alcohol?

Man 1: relax through drinking.

Woman 1: please learn how to protect yourself while men force you to drink. If you see a man coming late to our table, you have to force him to drink three cups. Therefore, man can get drunk easier than women do.

100623_001 June 2320101227 an hour and a half conversation with two men during lunch time in Fruit Garden Restaurant in Kaiping Two men, their age is between 25 and 26

Interviewer: More women drink in today's China?

Man A: Yes

Interviewer: Why? Would you explain to me a little bit?

Man A: Because China developed 
Man B: economic developed in society

Man A: human beings' living gets better in China...so not like women lived at home in the old days, women could come out and play

Interviewer: What do you mean by women could come out and play?

Man B: like women helped at home in the old days, men could come out and play (Hua Tian Jiu di)...but now women can also play outside too...

Man A: Today's China, men can stay home and help...

Man B: This is a developed society problem...it connected to economic perspectives

Interviewer: can you give me some examples?

Man B: in the old days, only men work outside, and only one income for one family. Men need to feed

family with finances...now people get wealthy so that they can think about alternative life joyful things

such as entertainments or interests - drinking alcohol nowadays. Men can earn money, so do women. Men could drink; women have the right to drink too...

Interviewer: What is your idea?

Man A: no idea. (He nodded his head)

Interviewer: What do you think of women expect through drink alcohol?

Man B: women drink in China ...there are many kinds ... drinking wine, women can be very high

cast...They drink because they only look for tastes of wine and keeping healthy. Some women in China

only like to be over drinking (Pei jiu) 䢃酒 this kind of the drinking entertainment is to buy drink, drank

and drunk.

Interviewer: how could be drink, drank and drunk?

Man B: I do not know how to say anything to you

Man B: The kind of Pei Jiu behavior is a Chinese wine culture. For example, I competed with you through drinking, and to see which one of us get drunk first. We also called this kind of drinking culture is "Dou

Jiu." 斗酒

Interviewer: how to compete?

Man B: through a simple dice game ...looser will drink. Through drinking, which one will drunk, he/she is a looser. Many kinds of drinking games, scissor, paper and rock or dice game. Hong Kong people have their game. Canton people also have their own drinking game... however, in Guang Dong province, dice game is the popular one for drinking game.

Man A: Beijing people only sing in KTV and order some drinks, they do not like our Guang Dong people passion within drinking

In Beijing, a group of friends would listen to one person singing in KTV, then, they would praise the singer with clapping their hands - Men B: "much healthier." If people in bars, they would choose to drink and dance, but no sing KTV like us Guang Dong people.

Man A: For men, they drink for passion. For example, through drinking alcohol, men can speak very openly and they can tell something in which they hide in mind... release and open to talk...

Man B: Women have many purposes to drink... we hardly recognize (many women many kinds of drinking)

Man B: for example, some women who drink because of they like the taste of alcohol, but some women like to pei jiu

Man A: for example, a group of women will not order beers while chatting, but would have red wine while chatting

Man A: oh, there is a passion; men would suggest drinking alcohol to increase the chatting motivation

Man B: women choose red wine to drink...except beers only for drinking competition in bars

Interviewer: What kinds of beer?

Man B: blue belt, Baiwei and Li Jia ...drinking brands? Only depended on the drinking fashion. If this season is towards to fashion blue belt, women will choose to drink blue belt

Man A: some would choose to drink western wine

Man B: western wine...you can add some juice to mix so that you can drink sweetly

Interviewer: why women drink wine in Kaiping?

Man A: because taste better

Man B: only two kinds in Kaiping: one kind is taste wine; the other kind is pi jiu.

Interviewer: have you seen young women drink spirit or distilled spirit?

Man B: not for young women? 
Interviewer: why?

Man B: Taste bitter (Lei Jiu)

Man B: young people will not choose this kind of drink to drink.

Interviewer: how about young men?

Man B: no they will not choose bitter wine to drink, because they do not want to get drunk easily. Only my opinion, young men would choose to taste better drink to drink because they need to keep chatting in a good mood.

Interviewer: why you say older people would choose to drink spirit more than young people?

Man A: spirit and distill spirit only for people who are good at drinking...for those who cannot drink well but still want to drink, they will not choose this kind of alcohol to drink

People can drink have a drinking alcohol habit (lao pai jui gui). The spirit and distill spirit, the ads are very boring ... for example, you and I have dinner, we order a spirit to drink, you maybe think we were very strange or wired.

Interviewer: I see... so young people choose to drink because of the attractive ads, right?

Man A and B: yes.

Man B: older men need sprit to come with dinner because they know this is healthier...young people will not suggest

Interviewer: how about women?

Man A: No! Women will not order spirit to come with dinner ... maybe they would choose to drink wine in a high cast restaurant

Man B: In Guang Dong, this kind wine culture becomes much more modern. While drinking, you cannot toast many people with a cup of drink; only the oldest one among a group of people will do so, to toast many people. ... Young people will toast older ones lower their cup; this is also a culture too. Young people will see the bottle if it is almost empty, and to add some more jiu... how to solve this problem?...put the bottle in the middle and let whoever wants to add jiu to their cups, they can help themselves. This is one way to respect elders. The alcohol tradition continues in today's culture. We are talking toasting is spirit or distill spirit. Pull spirit to a cup needs to be full, "tea half spirit full—Cha ban jiu man." Do not over flow the cup...please pull spirit within clock wise; only men will serve at the table...not women...if women sit at the same table, they will not speak often. If women are openness, they might serve others at the table to pull jiu to others.

Man A: Women drink wine is for happiness. If a person drinks happily at the table, you will drink with a passion. There is not any pressure.

Man A: for example, you are sad, the heart is very uncomfortable with a sad feeling...nervous...after drinking, you can release your emotion and become open to speak up whatever you want to say.

Man B: It is about women look for man to have intimacy relationship. Women will look for man in bars to have other kinds of needs with them (i.e. sex).

Man A: Drinking can reduce pressure...people need some attractive feelings in society...

July 20, 2010A conversation with a group of women in a coffee shop -Three highlights from them: 1) women who will not drink a normal brand of wine, 2) women in the general population who will not drink, and 3) women who can drink are not regular women. The reasons of women who drink alcohol were women wanted to show men their power and freedom/rights.

Women choose to drink differently based on attitude or drinking partner. For example, women might drink nothing for a particular partner, or might drink too much without getting drunk for a different partner.

There are a few highlights from interviewees' comments:

Interviewer: When you saw women drink, what do women expect through alcohol drinking?

Woman A and B:

1. Women who drink, who have high standards, who know how to taste and understand Jiu.

2. Women who drink, who are under a lot of pressure and may suffer depression, who need Jiu for release.

3. Women who drink, who have low self-efficacy and/or low morals, whose drinking lets them give up and enjoy playing. 
Woman C: "Most people who feel that these women are such poor people. I would say, she might drink too much because of sadness. Or some people will look down on women who get drunk, because they will think those women ruin their reputation. I would say these subjective comments on women drinking in bars are not accurate, because the people who talk about them do not understand these women's family backgrounds or histories. I am not a drinker, and do not like to drink either. However, sometimes I force myself to drink some because of my feelings. I do like the quote, (paraphrased) "Jiu is a kind of drink, but mixed with human feelings [the individual feeling of the person drinking], Jiu automatically illustrates multiple feelings [based on the feeling, Jiu becomes a different type of drink and allows human beings to experience something else]. Life is like a cup of Jiu, mix into a big crowd [a group of different people drinking together], and experiencing one another's feelings, thus, life becomes sour, sweet, bitter, and spicy."

Women D: If you are happy, cheer up with some friends; if you are sad, alcohol can give you a good night's sleep; if you want to express yourself, drinking alcohol can give you an excuse. The fact that more and more women are drinking in China relates to the complexity of society and human feelings and thoughts. Man A and B: Drinking because of life problems. People drink because of the loss of loved ones, or breaking up, or separating from friends, etc. I think drinking without harming others is a good expression. 